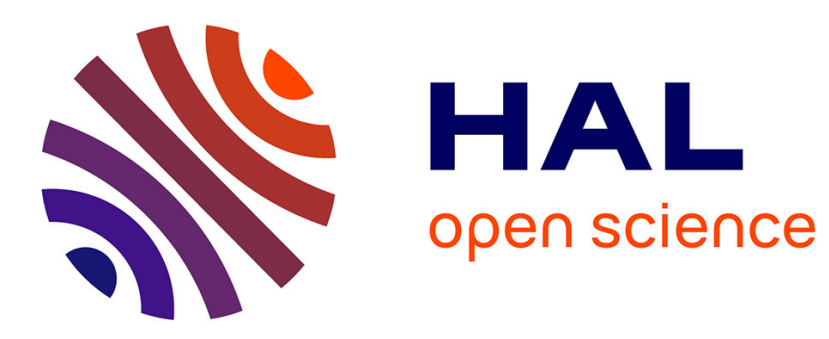

\title{
Intense ultrashort terahertz pulses: generation and applications
}

\author{
Matthias C Hoffmann, József András Fülöp
}

\section{To cite this version:}

Matthias C Hoffmann, József András Fülöp. Intense ultrashort terahertz pulses: generation and applications. Journal of Physics D: Applied Physics, 2011, 44 (8), pp.83001. 10.1088/00223727/44/8/083001 . hal-00597843

\section{HAL Id: hal-00597843 \\ https://hal.science/hal-00597843}

Submitted on 2 Jun 2011

HAL is a multi-disciplinary open access archive for the deposit and dissemination of scientific research documents, whether they are published or not. The documents may come from teaching and research institutions in France or abroad, or from public or private research centers.
L'archive ouverte pluridisciplinaire HAL, est destinée au dépôt et à la diffusion de documents scientifiques de niveau recherche, publiés ou non, émanant des établissements d'enseignement et de recherche français ou étrangers, des laboratoires publics ou privés. 


\title{
Intense ultrashort terahertz pulses: Generation and applications
}

\author{
Matthias C. Hoffmann ${ }^{1}$ and József András Fülöp ${ }^{2}$ \\ ${ }^{1}$ Max Planck Research Department for Structural Dynamics, University of Hamburg, CFEL, 22607 \\ Hamburg, Germany \\ ${ }^{2}$ Department of Experimental Physics, University of Pécs, Ifjúság ú. 6, 7624 Pécs, Hungary \\ matthias.c.hoffmann@mpsd.cfel.de,fulop@fizika.ttk.pte.hu
}

\section{Introduction}

The terahertz (THz) frequency range is usually defined as spanning two decades from 0.1 to $10 \mathrm{THz}$ or 3 to $300 \mathrm{~cm}^{-1}$. Also known as the far-infrared range, this part of the electromagnetic spectrum contains spectroscopic signatures for a multitude of physical phenomena, from small molecule rotational transitions used in astronomy [1] to the bandgap of superconductors [2]. This spectral region has received increased attention during the last two decades and a large amount of work has been published since then. However, the exploration of physical phenomena occurring in the THz region begun more than 110 years ago, starting with the discovery of reststrahlen bands in ionic salts by Rubens [3]. These studies resulted in improvements in measuring blackbody radiation and contributed directly to the formulation of Planck's law. An interesting historical overview of scientific investigations of the far infrared spectral region conducted in the last century can be found in Refs. $[3,4]$.

Spectroscopic work in the THz range has long been hindered by both the lack of strong sources and efficient detectors. Sandwiched between the readily accessible microwave frequency range $(<100 \mathrm{GHz})$ and the traditional infrared (IR) frequency range (>10 THz), the $\mathrm{THz}$ spectral region has long been 
described as the "THz-gap". Although a limited number of strong sources like gas lasers [5] have been available since the 1970's, this gap has only truly begun to close during the last two decades. Today a large variety of choices of tunable THz radiation sources are available such as Schottky diode mixers[6], quantum cascade lasers [7, 8], backward wave oscillators [9], p-germanium lasers [10, 11] as well as other interesting techniques [12] exist. Some very high average power continuous wave (cw) sources like nanosecond gas lasers [13] and free-electron lasers [14] have even enabled first studies of nonlinear effects in the THz range [15].

An important class of $\mathrm{THz}$ sources relies on the frequency conversion of femtosecond lasers to deliver coherent few-cycle THz pulses, enabling time-domain spectroscopy (TDS). While THz generation by nonlinear optical frequency conversion of picosecond pulses has been demonstrated already in the early 1970's [16], the field of THz TDS has undergone rapid technological development more recently with the widespread availability of femtosecond lasers, most notably the Ti:sapphire solid state laser. The use of femtosecond lasers enabled higher $\mathrm{THz}$ frequencies and conversion efficiencies previously unattainable from optically driven $\mathrm{THz}$ sources. Two main methods of generating ultrafast electromagnetic pulses with picosecond duration using femtosecond oscillators were established early on and are still the most widely used techniques: photoconductive switches [17, 18] and optical rectification [19]. Subsequently these techniques were adapted for amplified femtosecond laser systems for the generation of high intensity $\mathrm{THz}$ pulses which will be discussed in this review paper.

THz-TDS has the unique ability to characterize the complete electric field of a THz pulse to yield full phase and amplitude information, an advantage over typical detectors that measure only intensity. The complex dielectric function of a sample in the beam path can thus be determined directly [18] without having to rely on Kramers-Kronig relations. 
Ultrashort, near-single-cycle THz pulses can also be used to study dynamical processes by time-resolved pump-probe techniques. Here, a sample is excited by a femtosecond optical or near-IR pump pulse [20], causing changes in properties like the optical conductivity. A time-delayed THz pulse is then used as versatile probe in a broad range of $\mathrm{THz}$ frequencies. Because the complete $\mathrm{THz}$ field transmitted through or reflected from the sample can be measured directly, the real and imaginary parts of the optical conductivity can be determined and time resolution on the tens-of-femtoseconds scale can be achieved [21].

Conversely, strong THz fields can be used to excite samples that have resonances or other spectral features in the far-IR range. Promising results of nonlinear THz spectroscopy using single-cycle pulses exceeding field strengths of $100 \mathrm{kV} / \mathrm{cm}$ have recently been reported. Examples include acceleration of carriers such as free electrons in semiconductors [22, 23], and alignment of the induced dipole moment of nonpolar molecules [24]. The temporal profile of the electric field of the THz pulse can also be tailored to allow resonant pumping of collective excitations like phonons [25] or excitons [26].

This review provides an overview of various methods suitable for generating single- or few-cycle coherent THz pulses using ultrashort optical laser pulses. Recent technical developments in the generation of high intensity pulses that enabled the study of nonlinear phenomena in the THz range will be presented. Readers interested in the generation and detection of low energy, high repetition rate $\mathrm{THz}$ pulses and applications of quasi-static THz TDS are directed to Refs. [27-29].

The first sections of this review will discuss various techniques for ultrashort $\mathrm{THz}$ pulse generation. We will first discuss Tz pulse generation using photoconductive switches. Historically, this method was the first one to achieve single-cycle $\mathrm{THz}$ pulses with $\mu \mathrm{J}$-level pulse energies from an amplified femtosecond laser source [30]. An overview over broadband $\mathrm{THz}$ generation using gas plasmas will be given in Section 3. Methods based on nonlinear optical techniques like optical rectification and difference 
frequency generation will be detailed in Section 4. The recently developed method of tilting the intensity front of an optical pulse in order to optimize conversion efficiency through noncollinear optical rectification will also be discussed in this section. Finally, in Section 5 various applications of high field strength $\mathrm{THz}$ pulses in nonlinear $\mathrm{THz}$ spectroscopic experiments will be discussed.

\section{THz pulse generation by photoconductive switches}

\subsection{Concept of photoconductive switches}

The use of photoconductive switches is probably the most widely used method for the generation and detection of THz pulses in TDS. This technique was first introduced by Auston in the early 1980's [17]. Here, a biased semiconductor device, known as the Auston switch, is electrically shortened by a femtosecond laser pulse. Photoconductive switches consist of a semiconductor material with a short carrier lifetime (usually GaAs or silicon on sapphire, SoS) and an electrode structure composed of either gold or aluminum, with a gap on the order of $10 \mu \mathrm{m}$ (see Figure 1a). The electrodes are typically biased with a voltage of 10 to $50 \mathrm{~V}$, resulting in an electric field of a few $\mathrm{kV} / \mathrm{cm}$ across the switch. When the laser pulse hits the biased gap of the semiconductor, free carriers are created, and are accelerated by the bias field. The rapid change in polarization $P$ induced by the ultrafast acceleration of the carriers generates a sub-picosecond, single-cycle coherent electromagnetic pulse with a field strength of $E_{\mathrm{THz}} \propto \mathrm{d}^{2} \mathrm{P} / \mathrm{d} t^{2}$. This pulse propagates partially along the electrodes and is partially emitted into free space. The polarization ratio, defined as the ratio of $\mathrm{THz}$ field polarized in the direction of the bias field relative to that perpendicular to the bias field, is usually better than 100:1. 
(a)

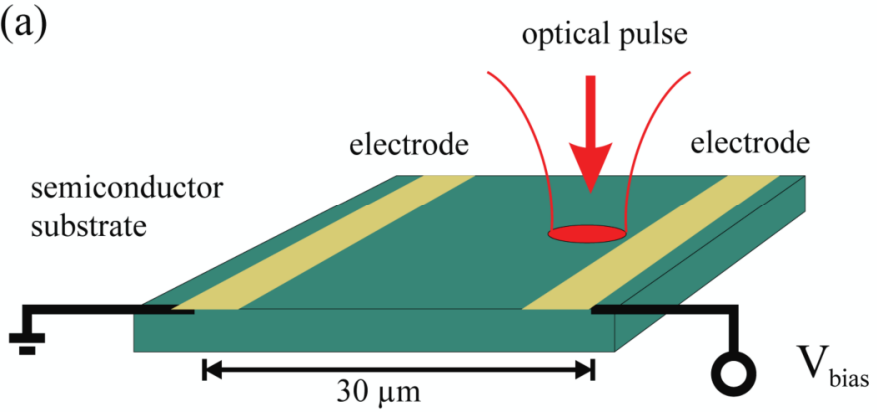

(b)

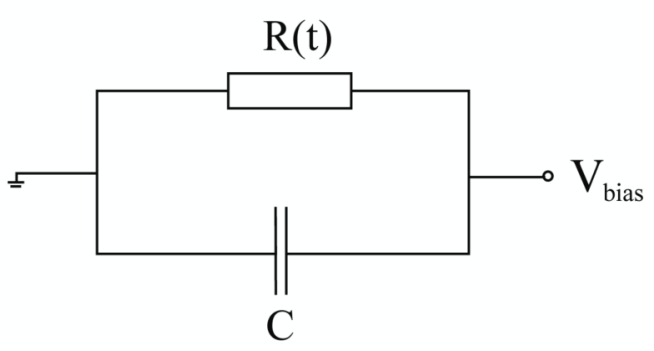

Figure 1. Scheme (a) and equivalent circuit (b) of a photoconductive switch.

The energy of the THz pulse stems from the release of the energy stored in the biased photoconductive switch, which can be viewed as a loaded capacitor with capacitance $C$ in parallel with a time-varying resistivity $R(t)$ (Figure 1b). The off-state of the photoconductive switch is characterized by high resistivity on the order of a few $\mathrm{M} \Omega$. Free carriers are liberated in the semiconductor at the gap between the metal electrodes by optical excitation with a short laser pulse. This instantly transforms the switch into its onstate as the resistance drops by several orders of magnitude. For typical dimensions of a GaAs-based switch, the gap capacitance can be estimated as $\mathrm{C}=\varepsilon \varepsilon_{0} A / d$, where $A$ is the area of the photoconductive gap and $d$ the penetration depth for $800 \mathrm{~nm}$ light, usually a few micrometers and $\varepsilon$ the dielectric constant of GaAs. The resulting capacitance is on the order of 10 to $100 \mathrm{fF}$. This low capacitance together with the low resistance of around $50 \Omega$ across the switch in its on-state result in circuit response times $R C$ on the order of a picosecond, leading to the emission of $\mathrm{THz}$ radiation by the antenna. The order of magnitude of the emitted THz pulse energy can be estimated from the electrostatic energy stored in the switch. This stored energy is the total amount of energy available for distribution between carrier kinetic energy and radiative energy, thereby placing an upper limit for the energy of the generated THz pulse. For a capacitance of $5 \mathrm{fF}$ and a bias voltage of $50 \mathrm{~V}$ we obtain a stored energy of $E_{\text {stored }}=(1 / 2) C V^{2}=10 \mathrm{pJ}$. Based on power measurements with bolometer or calibrated pyroelectric detectors, the typical $\mathrm{THz}$ pulse 
energies generated from photoconductive antennas are on the order of 1-10 fJ, thus only $0.1 \%$ of the stored capacitive energy is converted into free-space radiation.

\subsection{Material considerations}

The frequency response and conversion efficiency in THz generation with photoconductive switches depends crucially on the femtosecond pulse duration as well as on the choice of materials. The THz pulse radiated into free space is proportional to the second derivative of the transient current inside the switch. The rise time of this current is determined by the electric bias field applied to the switch, the pulse duration of the femtosecond laser and the carrier rise time intrinsic to the material. Obviously, the semiconducting material must be chosen so that the band gap is smaller than the photon energy of the laser. The material of choice for Ti:Sapphire based femtosecond lasers at $800 \mathrm{~nm}(1.55 \mathrm{eV})$ is gallium arsenide $(\mathrm{GaAs})$ with a band gap of $1.42 \mathrm{eV}$. This material has a fast carrier rise time and a high mobility, making it an excellent material for high efficiency photoconductive switches.

With better access to molecular beam epitaxy (MBE) facilities, LT-grown GaAs today is a common material for emitters as well as detectors. In the MBE growth process GaAs is deposited by molecular beam epitaxy at deliberately low temperature in order to cause an excess of arsenic in the material. By annealing the material, arsenic clusters are formed [31] which act as efficient traps for photo-induced carriers. Carrier lifetimes shorter than 500 fs can be achieved with this method [32-34]Similarly short carrier lifetimes can also be achieved with erbium nano islands embedded in GaAs [35]. Generally, there is a trade-off between optimally short carrier lifetimes and increased parasitic dark current leading to premature breakdown of the material. Very short carrier lifetimes are mostly important for the frequency response of $\mathrm{THz}$ detectors, for emitters the $\mathrm{THz}$ frequency spectrum is determined mostly by the laser pulse duration and the material absorption. The overall conversion efficiency of emitters fabricated from LT-GaAs and compensated semiinsulating GaAs with nanosecond carrier lifetime is comparable [36]. 
Historically the first material used was Silicon on Sapphire (SoS) [37]. This material consists of a fewmicrometer layer of amorphous silicon deposited on a sapphire substrate. The response of the material can be improved by radiation damaging through $\mathrm{O}^{2+}$ ions and is still widely used for $\mathrm{THz}$ detectors. The main advantages of SoS over other semiconductors like GaAs is the optically transparency and high dark resistivity.

Recently, new THz emitters have been developed that are able to work with femtosecond fiber lasers at 1.5 micron wavelength [38]. The emitters are based on photoconductive switches fabricated from lowtemperature-grown InGaAs/InAlAs multi-layer structures.

In addition, the available $\mathrm{THz}$ bandwidth is further limited in photoconductive switches by $\mathrm{THz}$ absorption and dispersion in the substrate. This is typically dominated by the phonon resonances of the semiconductor material for example by the TO phonon resonance at $7.56 \mathrm{THz}$ of GaAs. By utilizing the part of the THz pulse that is radiated into free space on the same side of the antenna substrate as the incident laser pulse this problem can be partially mitigated since propagation through the substrate is eliminated [39].

\subsection{Antenna geometry}

Several emitter antenna geometries other than the Auston switch have been evaluated, including the bowtie antenna, interdigitated structures, spiral antennas and other more sophisticated designs derived from microwave theory [40]. Surprisingly, the simple sliding contact structure shown in Figure 1a has been demonstrated to generate $\mathrm{THz}$ pulses with broad spectra up to $5 \mathrm{THz}$ and relatively high field strengths. The THz field can be enhanced by focusing the femtosecond laser close to the anode of the photoconductive switch structure, taking advantage of the locally trap-enhanced field at the anode [41]. 
For generation of $\mathrm{cw} \mathrm{THz}$ radiation through photomixing of two $\mathrm{cw}$ diode lasers at slightly different wavelength [42] spiral antennas are preferable. For further information regarding antenna structures, readers are referred to the reviews by Sakai and Mittleman [40, 29]

\subsection{High field pulses using large area emitters}

Using the miniaturized photoconductive switches described in the preceding section, with femtosecond oscillators ( $<5 \mathrm{~nJ} /$ pulse), fJ THz pulse energies, and field strengths in the range of 10 to $100 \mathrm{~V} / \mathrm{cm}$, can be achieved. The use of an amplified femtosecond laser system allows the same generation principle described earlier to be scaled up through enlarging the photoconductive antenna gap to a dimension on the order of $1 \mathrm{~cm}$ and increasing the bias voltage to several $\mathrm{kV}$. In an early demonstration of this technique, You et al have achieved $0.8 \mu \mathrm{J} \mathrm{THz}$ pulse energies at $10 \mathrm{~Hz}$ repetition rate using semi-insulating GaAs wafers with silver paint electrodes and pulsed bias voltages applied by a spark plug [30]. The maximum

$\mathrm{THz}$ energies were achieved with an external bias field of $10.7 \mathrm{kV} / \mathrm{cm}$ and laser fluencies of $0.1 \mathrm{~mJ} / \mathrm{cm}^{2}$. A quadratic dependence of the $\mathrm{THz}$ output power on the applied bias field and strong saturation at laser fluencies larger than $50 \mu \mathrm{J} / \mathrm{cm}^{2}$ were observed [43] and described by modeling for different materials. The resulting strong THz fields have been applied to achieve field ionization of Rydberg atoms [44].

However, the high voltages and the associated thermal load at higher repetition rates have prevented this method from finding more widespread usage. Further, large-aperture $\mathrm{THz}$ emitters typically exhibit $\mathrm{THz}$ spectra centered at relatively low frequencies. This is usually attributed to a lower acceleration field and the large distance between the electrodes. 

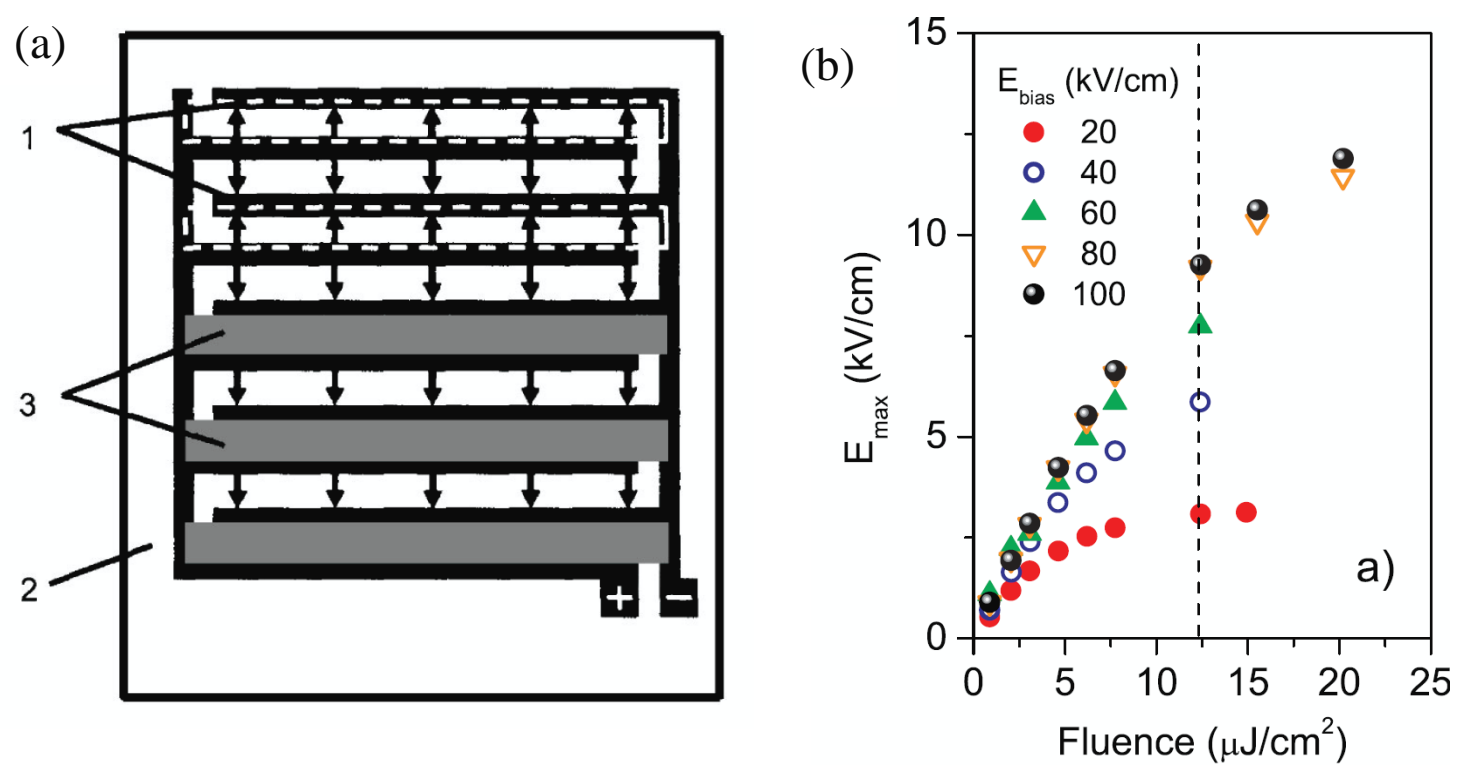

Figure 2 (a) Schematics of the THz emitting MSM. 1: interdigitated finger electrodes, 2: SI GaAs substrate, 3: opaque metallization shadowing one electric field direction. The electric field direction is indicated by arrows [45]. (b) peak THz electric field as a function of excitation fluence on the photoconductor for different acceleration fields at $250 \mathrm{kHz}$ repetition rate [46].

To circumvent the problem of a large thermal load, Dreyhaupt et al. have developed large area interdigitated metal-semiconductor-metal (MSM) structures[45, 47]. Here, metal electrodes with $5 \mu \mathrm{m}$ width and spacing are fabricated on a semi-insulating GaAs substrate with a $1 \mu \mathrm{m}$ thickness $\mathrm{Si}_{3} \mathrm{~N}_{4}$ insulation layer (Figure 2). A further metallization layer covers every other finger electrode spacing in order to prevent destructive interference of the generated $\mathrm{THz}$ field. These structures can be operated with an external bias voltage of less than $50 \mathrm{~V}$ and can achieve $\mathrm{THz}$ to optical pulse energy ratios of up to $5 \times 10^{-4}$ using $\mathrm{nJ}$ pulses from a femtosecond oscillator. This method can be scaled up further using amplified laser systems with larger aperture antennas with an active area up to $10 \times 10 \mathrm{~mm}^{2}$. Using pump pulse energies of $4 \mu \mathrm{J}$, THz electric fields up to $36 \mathrm{kV} / \mathrm{cm}$ at repetition rates of $250 \mathrm{kHz}$ can be obtained 
[46] In these experiments very high THz to optical pulse energy ratios of $2 \times 10^{-3}$ were achieved at a fluence of $20 \mu \mathrm{J} / \mathrm{cm}^{2}$ and bias voltage in the sample of $100 \mathrm{kV} / \mathrm{cm}$.

In conclusion, photoconductive switches can be used for highly efficient $\mathrm{THz}$ generation since the energy of the THz pulse stems from the applied bias field. This compares favorably to optical rectification for small pump pulse energies [48]. Strong saturation however limits scaling with increasing laser pulse energy. For high repetition rate, low pulse-power laser systems they provide an effective source for $\mathrm{THz}$ single cycle pulses with frequency bandwidth of less than $5 \mathrm{THz}$. 


\section{Terahertz generation and detection in gas plasmas}

A promising technique to obtain single-cycle $\mathrm{THz}$ pulses with high field strengths using femtosecond lasers is the generation of $\mathrm{THz}$ pulses by nonlinear processes in gas plasmas. $\mathrm{THz}$ generation in plasmas has the advantages that there is no damage threshold for the emitter since the gas target is continuously replenishable and that the available bandwidth of the THz pulses is essentially limited only by the duration of the pump laser pulse since no material absorption like optical phonons need to be considered. Conversely, THz pulses can also be detected in gases using THz-induced second harmonic generation of fluorescence. In this section we will briefly discuss plasma-based $\mathrm{THz}$ generation and detection methods with emphasis on recent developments. More in-depth reviews of these topics can be found in Refs [49, $50]$.

\subsection{Terahertz generation in gas plasmas}

When a femtosecond laser pulse is focused in gas, a plasma is generated when a threshold intensity on the order of $\mathrm{TW} / \mathrm{cm}^{2}$ is exceeded. This plasma emits a broad continuum of coherent and incoherent radiation. Using laser plasmas to generate coherent far-infrared radiation at THz frequencies was first proposed and demonstrated by Hamster et al. [51] in 1993 with TW-level lasers. A significant improvement in efficiency over this approach was achieved in 2000 by Cook and coworkers [52] through mixing of femtosecond pulses with center frequency $\omega$ with their second harmonic at $2 \omega$ within the gas plasma, yielding generation efficiency increased by orders of magnitude. In 2007, Kim et al. [53] reported on pump-to-THz conversion efficiencies exceeding $1 \times 10^{-4}$ and pulse energies up to $5 \mu \mathrm{J}$. The generation of ultrabroadband $\mathrm{THz}$ pulses containing frequencies up to $100 \mathrm{THz}$ has been demonstrated very recently by Thomson by using 19 fs laser pulses [54] and carefully optimized BBO crystal angles.. 
In the most common experimental realization (Figure 3), a 800-nm femtosecond laser pulse generated by an amplified Ti:sapphire laser with a pulse energy of $0.5 \mathrm{~mJ}$ or more is focused by a spherical lens into a gas target to form a plasma. Before the focus, a second harmonic generation (SHG) crystal, typically beta barium borate, $(\beta-\mathrm{BBO})$, is inserted to produce $400 \mathrm{~nm}$ wavelength light. Both the fundamental and the second harmonic wavelengths are mixed in the plasma. The THz generation process is sensitive to the relative phase between the fundamental and the second harmonic fields. This can be adjusted either by translating the SHG crystal utilizing the dispersion of the gas or by using a phase shifting glass plate. In case of the frequently used type I SHG the polarization of the $400 \mathrm{~nm}$ light is mostly perpendicular to that of the fundamental. The relative electric field polarization directions can be controlled by a dual-band waveplate inserted between the SHG crystal and the focus to set them, e.g., parallel [55]. The accumulated temporal retardation of the SHG pulse with respect to the fundamental pulse due to material dispersion can be (pre)compensated by a birefringent plate such as $\alpha$-BBO or calcite.

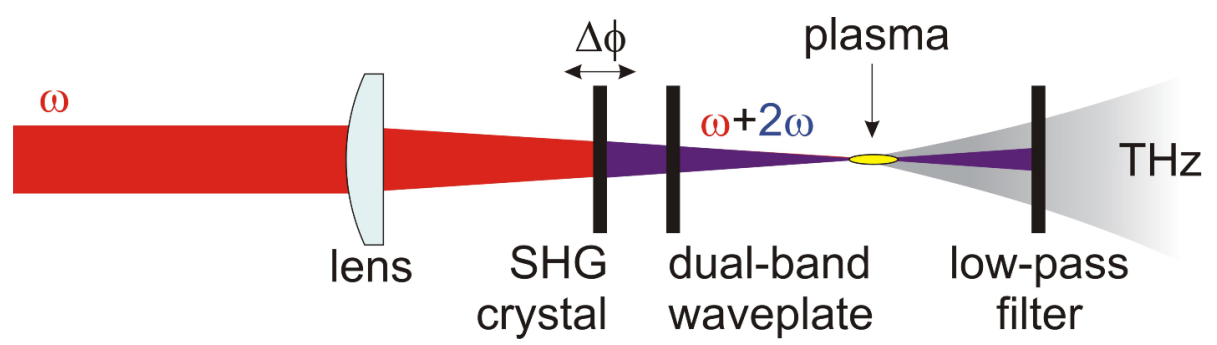

Figure 3. Scheme of the experimental realization of the two-color THz generation in plasma gas targets. $\Delta \phi$ indicates the relative phase between the fundamental and its second harmonic, which can be varied e.g. by translating the SHG crystal. 
As the gas target for plasma generation ambient air, nitrogen or some type of noble gas is usually used. Although it is convenient to use air, the absorption of water vapour reduces the THz yield. By using noble gases with higher tunnel ionization rates ( $\mathrm{Kr}$ or $\mathrm{Xe}$ ), the $\mathrm{THz}$ generation efficiency can be significantly improved [53]. The pressure of the target gas also strongly influences the THz generation efficiency. With low pump pulse energies $(850 \mu \mathrm{J})$ after a linear increase in $\mathrm{THz}$ emission up to 0.1 bar, a relatively constant signal was measured in the pressure range of 0.1-2 bar [49]. On further increasing the pressure, the signal drops rapidly due to collision of accelerated electrons with molecules. In contrast to this, at multi-mJ pump energies saturation was observed at higher pump fluences [53]. A systematic study of the $\mathrm{THz}$ output energy and spectral content for varied pump energy (up to $6 \mathrm{~mJ}$ ) and pressure of different gas targets has recently been reported.[56]. They observed a significant two-color phasing effect for all gases, and saturation only for the heavier gases ( $\mathrm{Kr}$ and $\mathrm{Xe})$.

\subsection{Mechanism of THz generation}

To describe the rather complex phenomenon of $\mathrm{THz}$ generation in plasma driven by a two-color laser field, first a perturbative model based on four-wave frequency mixing, a third-order nonlinear optical process, was proposed by Cook et al [52]. However, the third order nonlinearity resulting from either bound electrons or free electrons in the plasma due to ponderomotive or thermal effects is too small to explain the observed THz field strengths [57]. Further, the existence of an intensity threshold for $\mathrm{THz}$ generation was observed. In order to account for these effects a semiclassical transient photocurrent model was used later $[58,59,56]$, where the $\mathrm{THz}$ radiation is described as a result of the unidirectional motion of electrons (giving a current surge) during tunneling ionization by the symmetry-broken twocolor laser field. In the strong external electric field of the focused femtosecond laser pulse applied to the atom, the atomic Coulomb potential is distorted and there is a finite probability that the electron will 
tunnel through the potential barrier. The resulting free electrons and the positively charged ions form a plasma. The free electrons are then further accelerated by the applied field emitting THz radiation.

Recently, a two-step model has been developed [50,60], which provides an even more detailed insight into the THz generation process. The first step, treated fully quantum mechanically by numerically solving the time-dependent Schrödinger equation, is asymmetric gas ionization by the two-color laser field (Figure 4). The second step, treated analytically, describes the interaction of the ionized electron wavepackets with the surrounding medium. Scattering of the ionized electrons by the atoms of the medium disrupts their original trajectories, which results in a coherent bremsstrahlung echo at $\mathrm{THz}$ frequencies. Generally, the full treatment of spatio-temporal propagation in the plasma is quite complex and needs to take into account effects such as plasma-induced phase modulation and defocusing [61]. For a complete description of $\mathrm{THz}$ generation in gas plasmas highly sophisticated simulations will be required. 


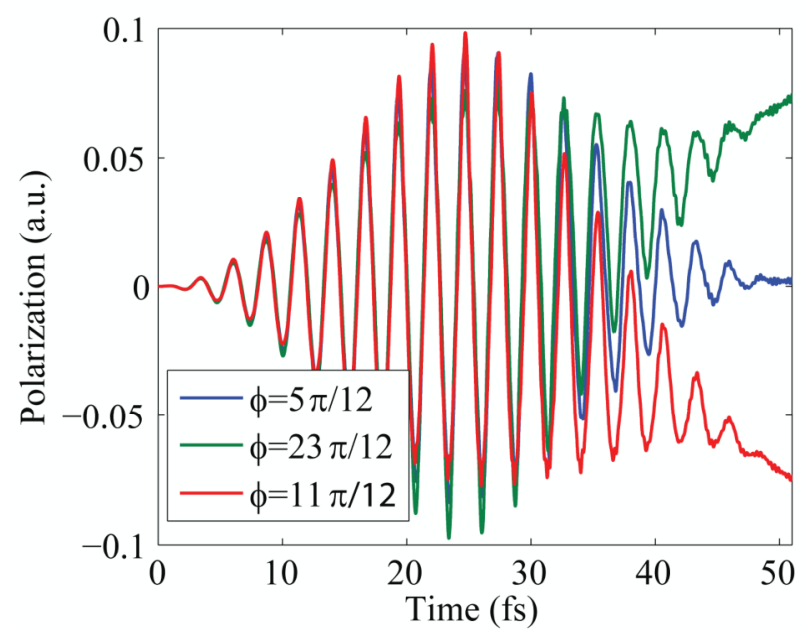

Figure 4:Time dependent polarization for Ar atoms subjected to $800 \mathrm{~nm}+400 \mathrm{~nm}$ optical pulses with various values of the relative phase $\phi$ between the two components. Asymmetric polarization occurs for values of $\phi$ close to integer multiples of $\pi$. After Ref. [50], used with author's permission.

\subsection{Detection of $\mathrm{THz}$ waves in gases}

The nonlinear interaction between a laser field with frequency $\omega$ and a THz field in gas can lead to the generation of the second harmonic of the laser field at $2 \omega$ frequency. Unlike THz generation in gases, this process can be well described by a four-wave mixing model [50]. As shown in Refs. [62, 63] generation of the second-harmonic field by the four-wave mixing process can be used to measure the temporal dependence of the THz electric field. In the detection stage (Figure 5) both the THz and the probe beam with frequency $\omega$ are focused between two needle or plate shaped electrodes having a small separation of the order of $1 \mathrm{~mm}$ [63]. The intensity is kept just below the plasma formation threshold. A low-frequency AC bias field with peak electric field strength on the order of $10 \mathrm{kV} / \mathrm{cm}$ is applied to the electrodes synchronized to the laser pulse train. The role of the AC bias field is to introduce a cross-term in the 
second harmonic signal $I_{2 \omega} \propto\left(\chi^{(3)} I_{\omega}\right)^{2} E_{\mathrm{AC}} E_{\mathrm{THz}}$, which is modulated by the same AC frequency. This results in a heterodyne process that allows only this coherent cross-term to be detected through lock-in amplification at the AC frequency. By changing the delay between the optical pulse and the THz pulse the temporal shape of the THz field can be recorded. With this detection technique THz TDS has been demonstrated covering the frequency range from 0.3 to $15 \mathrm{THz}$ [63] and even higher bandwidths can be reached with shorter laser pulses. Absorption gaps in the detectable spectra between 5 and $10 \mathrm{THz}$ caused by phonon resonances in materials commonly used for electro-optic sampling are circumvented by the use of this technique. The detection of $\mathrm{THz}$ waves in gases has recently been extended to $\mathrm{THz}$ remote sensing by coherently manipulating the (UV) fluorescence emission from an asymmetrically ionized gas plasma [64].

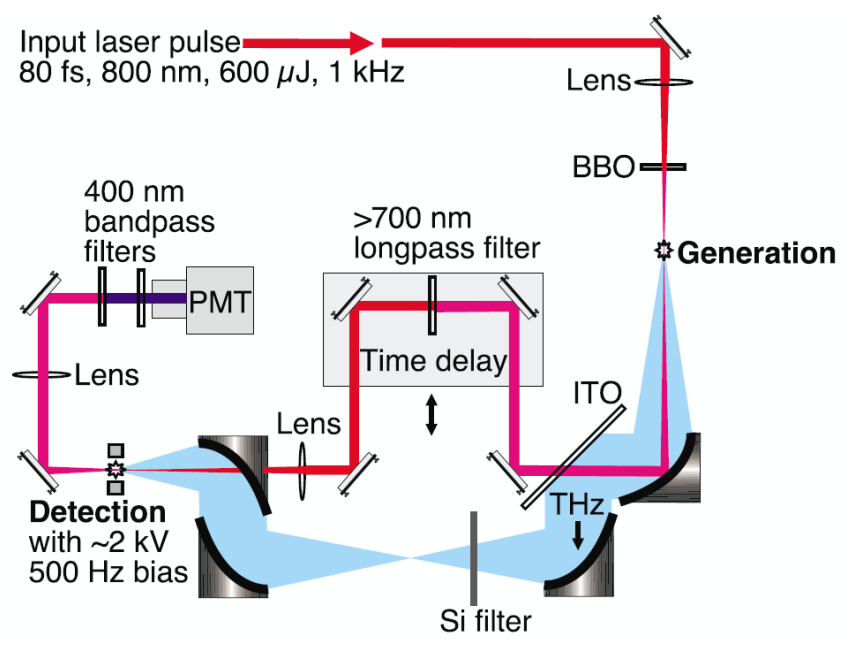

Figure 5. Schematic diagram of the experimental setup for coherent $\mathrm{THz}$ generation and detection in plasmas (from [63], used with permission)

In conclusion, generation and detection of $\mathrm{THz}$ pulses in gas plasmas is a rapidly developing technique for ultrafast spectroscopy covering the entire $\mathrm{THz}$ range well into the mid-infrared. Because phase 
mismatch due to phonon absorption present in electro-optic crystals can be circumvented by detection in gases, the undistorted shape of the THz pulse generated in the plasma can be measured, preserving the full bandwidth over an extremely large spectral range.

\section{THz pulse generation by optical rectification}

Another widely used method for ultrashort THz pulse generation is optical rectification of femtosecond laser pulses. Optical rectification (OR) was first demonstrated in KDP and K*DP crystals in 1962 by Bass et al. [65] using 100-ns pulses from a ruby laser at $694 \mathrm{~nm}$. Far-infrared generation using OR of picosecond pulses in $\mathrm{LiNbO}_{3}$ has been predicted [16] and demonstrated [66] as early as 1971. Free-space radiation with a bandwidth of $1 \mathrm{THz}$ by OR of colliding-pulse mode-locked (CPM) dye laser pulses was generated and detected in the time domain by Hu et al. [67].

\subsection{Theory and materials}

OR is a second-order nonlinear optical process, which is based on difference-frequency generation (DFG) between the spectral components of a single femtosecond pump pulse (intrapulse DFG). The nonlinear polarization induced by the pump pulse can be calculated as [68]

$$
P_{\mathrm{NL}}(\Omega)=\varepsilon_{0} \chi^{(2)} \int_{0}^{\infty} E(\omega+\Omega) E^{*}(\omega) d \omega
$$

where $\varepsilon_{0}$ is the permittivity of free space, $\chi^{(2)}$ is the second-order nonlinear susceptibility of the medium, $E(\omega)$ is the one-sided ( $\omega>0$ ) Fourier-component of the pump pulse, $\omega$ is the optical frequency, and $\Omega$ is the THz frequency. According to Eq. (1), a narrowband pump pulse generates only 
low $\mathrm{THz}$ frequencies, while a broadband optical pulse can generate a broad $\mathrm{THz}$ spectrum containing also higher THz frequencies.

Besides the nonlinear polarization, the buildup of the THz field over macroscopic distances in the medium is also influenced by the dispersion of the medium. The efficiency of OR is highest when the phase matching condition [69]

$$
\Delta \mathbf{k}(\Omega)=\mathbf{k}(\Omega)+\mathbf{k}(\omega)-\mathbf{k}(\omega+\Omega)=0
$$

is fulfilled. Since $\Omega<<\omega$ for OR, the term $\mathbf{k}(\omega+\Omega)-\mathbf{k}(\omega)$ can be approximated by $\partial k /\left.\partial \omega\right|_{\omega_{0}} \cdot \Omega$, which gives $\Delta k=\left[n(\Omega)-n_{\mathrm{g}}\left(\omega_{0}\right)\right] \Omega / c$ in the case of collinear THz generation. Here $\omega_{0}$ is the mean pump frequency, $c$ is the speed of light in vacuum, $n$ and $n_{\mathrm{g}}$ are the refractive index and the group index, respectively. Thus, phase matching can also be formulated as matching the $v(\Omega)$ phase velocity of the generated $\mathrm{THz}$ radiation to the group velocity $v_{\mathrm{g}}\left(\omega_{0}\right)$ of the optical pump pulse:

$$
v(\Omega)=v_{\mathrm{g}}\left(\omega_{0}\right) .
$$

In case of velocity matching and negligible dispersion in the $\mathrm{THz}$ range the output is a single $\mathrm{THz}$ pulse with its electric field shaped as the time derivative of the optical pump pulse envelope [70]. For Gaussianlike pump pulse envelopes this means that single-cycle $\mathrm{THz}$ pulses can be generated.

For phase matched conditions, the efficiency of DFG by long plane wave pulses in the absence of pump absorption or depletion, taking into account THz absorption can be described by [69]

$$
\eta_{\mathrm{THz}}=\frac{2 \Omega^{2} d_{\mathrm{eff}}^{2} L^{2} I}{\varepsilon_{0} n_{\mathrm{NIR}}^{2} n_{\mathrm{THz}} c^{3}} \cdot \exp \left[-\alpha_{\mathrm{THz}} L / 2\right] \cdot \frac{\sinh ^{2}\left[\alpha_{\mathrm{THz}} L / 4\right]}{\left[\alpha_{\mathrm{THz}} L / 4\right]^{2}},
$$


where $d_{\text {eff }}$ is the effective nonlinear coefficient, $L$ is the material length, $I$ is the pump pulse intensity, $\alpha_{T H z}$ is the absorption coefficient in the material at $\mathrm{THz}$ frequencies, $n_{\mathrm{NIR}}$ and $n_{\mathrm{THz}}$ are the refractive indices at the pump and THz frequencies, respectively. Eq. (4) can be used to compare the suitability of different nonlinear optical crystals for OR by defining a figure of merit (FOM) defined as [71].

$$
\begin{aligned}
& F O M_{N A}=\frac{\mathrm{d}_{\text {eff }}^{2} \mathrm{~L}^{2}}{n_{N I R}^{2} n_{T H z}} \\
& F O M_{A}=\frac{4 \mathrm{~d}_{\mathrm{eff}}^{2}}{n_{N I R}^{2} n_{T H z} \alpha_{T H z}}
\end{aligned}
$$

Here Eqn 5a is valid for negligible THz absorption in the generation crystal and Eqn $5 \mathrm{~b}$ is valid in the case of significant absorption. The most relevant parameters of materials frequently used for OR are shown in Table 1. As can be seen in the Table, at room temperature DAST [72] an organic material, has the highest FOM value. An increase the damage threshold of DAST to a value of $2.8 \mathrm{GW} / \mathrm{cm}^{2}$ through annealing was recently reported [73], however its use for high-energy $\mathrm{THz}$ pulse generation yet needs to be demonstrated. We note that there are also other promising organic materials with very large effective nonlinearities, such as BNA [74] or OH1 [75]. CdTe has the third highest FOM value at room temperature, but it is strongly absorbing at $800 \mathrm{~nm}$, the wavelength of Ti:sapphire lasers which are the most widely used pump sources for $\mathrm{THz}$ generation. The most commonly used material for OR is ZnTe, which has the fourth highest FOM value. Its main advantage is that velocity matching is approximately fulfilled in collinear geometry for Ti:sapphire pumping and far-infrared radiation of a few $\mathrm{THz}$ frequency. However, ZnTe has strong two-photon absorption at $800 \mathrm{~nm}$, which leads to the generation of free carriers and, in turn, to increased THz absorption (see also Sec. 4.3). Hence, the useful pump intensity is limited, which is a serious restriction in high-energy $\mathrm{THz}$ pulse generation [76]. The situation is similar with $\mathrm{GaP}$, another semiconductor material frequently used for OR with considerably smaller FOM value. 
Velocity matching is approximately fulfilled in collinear geometry for about $1 \mu \mathrm{m}$ pump wavelength, suitable to be pumped by compact Yb-based solid-state laser sources, but two-photon absorption limits the usable pump intensity.

\begin{tabular}{|l|l|l|l|l|l|l|}
\hline Material & $\begin{array}{l}d_{\text {eff }} \\
(\mathrm{pm} / \mathrm{V})\end{array}$ & $n_{800 \mathrm{~nm}}^{g r}$ & $n_{T H z}$ & $n_{1.55 \mu m}^{g r}$ & $\begin{array}{l}\alpha_{T H z} \\
\left(\mathrm{~cm}^{-1}\right)\end{array}$ & FOM \\
$\left(\mathrm{pm}^{2} \mathrm{~cm}^{2} / \mathrm{V}^{2}\right)$
\end{tabular}

Table 1. (after [71]) Properties of a few materials suitable for OR. Except for DAST, the THz index $n_{\mathrm{THz}}$ and absorption coefficient $\alpha_{\mathrm{THz}}$ are given for $1 \mathrm{THz}$. For DAST these values are given for $0.8 \mathrm{THz}$. All values are for room temperature except those for stochiometric $\mathrm{LiNbO}_{3}(\mathrm{sLN})$ at $100 \mathrm{~K}$.

GaSe has a moderate FOM value, but it has the advantage that velocity matching is possible based on birefringence, in contrast to the other semiconductors in Table 1. Difference frequency generation in 
GaSe between two near-infrared pulses generated by the signal and idler of an optical parametric amplifier (OPA) yields tunable pulses covering the entire mid-infrared from $4 \mu \mathrm{m}$ to $20 \mu \mathrm{m}[77,78]$. This principle can be modified to generate carrier-envelope phase stable mid-IR pulses by using the signal pulses from two OPAs derived from the same white light seed. Recently, focused electric field strengths as high as $100 \mathrm{MV} / \mathrm{cm}$ were demonstrated around $50 \mathrm{THz}$ frequency. It should be noted that the obtainable field strengths scale strongly with the THz frequency (see Eq. (4)), hence, in reaching such high field strengths the high THz frequency (corresponding to $6 \mu \mathrm{m}$ wavelength) was essential. Besides the increased conversion efficiency for shorter THz wavelengths (Eqn. 4) a key issue is that the reachable focus diameter scales linearly with wavelength, resulting in linear scaling of the electric field with wavelength. These intense mid-IR pulses can be further stabilized for long-term drift [79] and used for pump-probe measurements sensitive to the absolute optical phase.

Recently, stochiometric $\mathrm{LiNbO}_{3}(\mathrm{sLN}$ ) became a widely used material for OR. In LN, THz electromagnetic waves are strongly coupled to highly polar, "soft" optic phonons associated with ferroelectric phase transition, yielding mixed phonon-polariton modes [80]. The polarizabilities of these modes give rise to many of the favorable nonlinear optical properties of lithium niobate (LN). Besides its large effective nonlinearity resulting in the second highest FOM value in Table 1, LN has a bandgap much larger than that of semiconductors allowing only three-photon absorption to be effective at $800 \mathrm{~nm}$ pumping. This allows for high pump intensities, essential for high-energy $\mathrm{THz}$ pulse generation. As can be seen in Table 1, the THz refractive index for LN is significantly larger than the optical group index, and therefore no collinear velocity matching is possible. Quasi-phase matching in periodically poled LN can be used to enhance conversion efficiency, resulting in multi-cycle $\mathrm{THz}$ pulses $[81,68]$ with a fixed number of cycles. In bulk LN, generation of single cycle THz pulses with high conversion efficiency can 
be achieved by the tilted-pulse-front pumping technique with noncollinear velocity matching described in the following section.

\subsection{Velocity matching by tilting the pump pulse front}

Tilted-pulse-front pumping (TPFP) was proposed and demonstrated by Hebling et al. in 2002 for efficient phase-matched $\mathrm{THz}$ pulse generation in $\mathrm{LN}$ [82]. In this technique, the $\mathrm{THz}$ radiation generated by the tilted pulse front of the pump propagates perpendicularly to this front (Figure 6) with the THz phase velocity $v(\Omega)$, according to Huygens' principle. This leads to a non-collinear propagation geometry, where the angle between the propagation directions of the $\mathrm{THz}$ radiation and the pump pulse is equal to the tilt angle $\gamma$ of the pump pulse front relative to the pump phase fronts (which is perpendicular to the pump propagation direction). The $\mathrm{THz}$ wave propagates with a fixed phase relative to the pump pulse front if the following velocity matching condition is fulfilled:

$v_{\mathrm{g}}\left(\omega_{0}\right) \cos \gamma=v(\Omega)$.

In the case of LN where $v_{\mathrm{g}}\left(\omega_{0}\right) \geq v(\Omega)$, or equivalently $n_{\mathrm{g}}\left(\omega_{0}\right) \leq n(\Omega)$, Eq. (6) can be fulfilled by choosing an appropriate pulse front tilt angle $\gamma$. We note that the velocity-matching condition given by Eq. (6) is equivalent to a non-collinear phase-matching condition according to Eq. (2) [71]. To show this one needs to take into account the relation $[83,84]$

$\tan \gamma=\left.\left[n\left(\omega_{0}\right) / n_{\mathrm{g}}\left(\omega_{0}\right)\right] \omega_{0}(\mathrm{~d} \varepsilon / \mathrm{d} \omega)\right|_{\omega_{0}}$,

which links pulse-front tilt and angular dispersion $\left.(\mathrm{d} \varepsilon / \mathrm{d} \omega)\right|_{\omega_{0}}$. Here $\varepsilon$ is the angle of deviation between an incoming and outgoing ray introduced by a dispersive element such as a prism or a grating. 


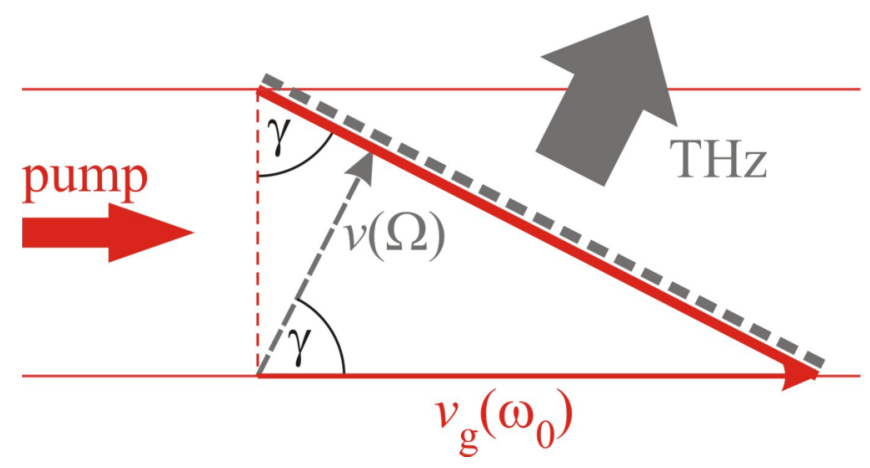

Figure 6 Velocity matching using TPFP. The thick red/solid line indicates the pump pulse front, the thick grey/dashed line indicates the THz phase front. The arrows indicate the propagations direction and velocities of these two surfaces.

In the Cherenkov geometry (Fig. 7), which was first used for THz generation by OR $[19,85,86]$, THz radiation from bulk $\mathrm{LN}$ or $\mathrm{LiTaO}_{3}$ is generated by OR of a femtosecond laser pulse with a tight point or line focus. The $\mathrm{T} \theta_{\mathrm{C}}$. Here, the same condition is obtained for the angle $\theta_{\mathrm{C}}$ as that given by Eq. (6) for the pulse front tilt angle $\gamma$, therefore the two angles are equal. However, the conical THz emission geometry is disadvantageous for applications due to practical issues with collimating and guiding the $\mathrm{THz}$ radiation. Further, the Cherenkov geometry does not allow the use of an extended pump spot [85]. In Ref. [87] a series of spatially and temporally shifted focused pump pulse replicas were used for coherent control of the generated THz-frequency lattice waves. However because $\mathrm{THz}$ damping occurs between as well as within the irradiated regions, this approach yields considerably lower overall $\mathrm{THz}$ conversion efficiency than TPFP. In contrast, the TPFP technique allows using an extended pump spot, thereby enabling highenergy THz pulse generation. 


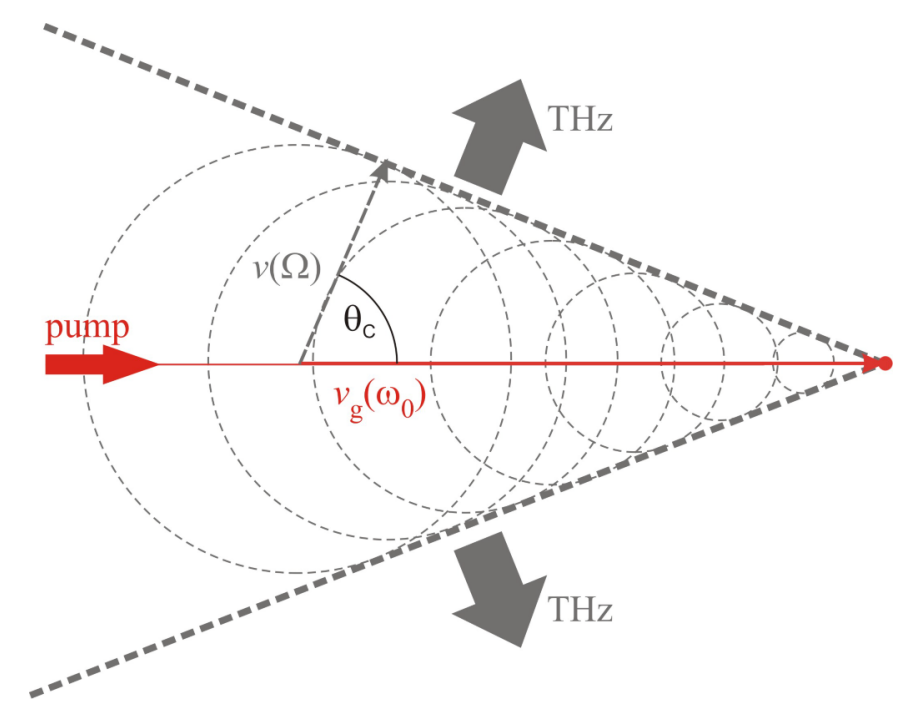

Figure 7 Cherenkov-like cone of emitted radiation due to OR of a subpicosecond optical pulse moving at a velocity $v_{\mathrm{g}}\left(\omega_{0}\right)$, which is greater than the THz phase velocity $v(\Omega)$, of the emitted radiation. After Ref. [86].

Due to the large difference between $n(\Omega)$ and $n_{\mathrm{g}}\left(\omega_{0}\right)$, TPFP in LN requires a pulse front tilt as large as $63^{\circ}-65^{\circ}$. In order to provide such large tilt angle (and the corresponding large angular dispersion, cf. Eqn. (7)) a pulse-front-tilting setup consisting of a grating and a lens is used in experiments (Figure 8). The angular dispersion introduced by the grating is further enlarged by the lens, which also recreates the original short pulse duration inside the medium by imaging the grating surface into the crystal.. Additionally, a half wave plate is used to match the polarization of the pulse to the z-axis of the LN crystal. The generated $\mathrm{THz}$ polariton wave is coupled out into free space through the surface of the crystal cut at the tilt angle $\gamma$. 
(a)

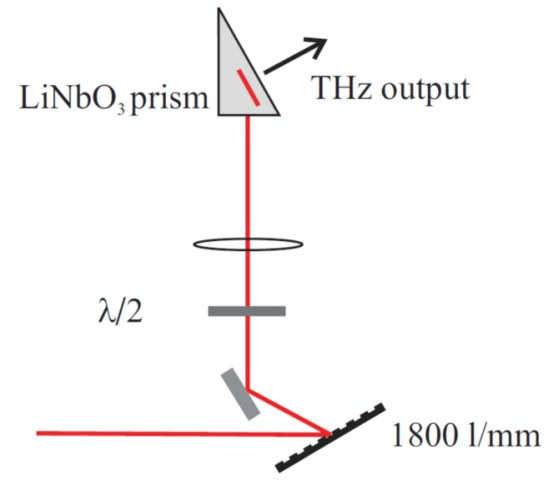

(b)

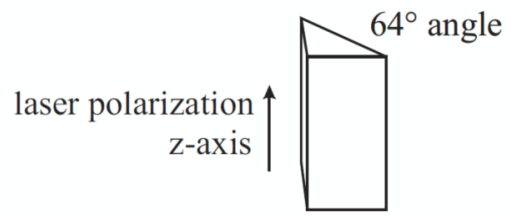

Figure 8 (a) Experimental setup for THz generation by TPFP in LN. (b) LN crystal orientation.

By changing the grating and imaging parameters, the experimental setup can easily be adapted to different pump wavelengths and repetition rates (Table 2). For example, TPFP with sLN at $1033 \mathrm{~nm}$ wavelength was demonstrated in Ref. [88] using an Yb-doped amplified fiber laser system. Here, repetition rates up to $1 \mathrm{MHz}$ and pump pulse energies in the $10-\mu \mathrm{J}$ range were employed. A reliable source of broadband $\mathrm{THz}$ pulses with high average power at $\mathrm{MHz}$ repetition rates is a prerequisite for applications in rapid $\mathrm{THz}$ spectroscopic imaging, high throughput screening, and signal processing. The THz output frequency in TPFP can be tuned by changing the pulse front tilt angle [67] in TPFP. This is possible by choosing a tilt angle matched to the polariton dispersion curve of the bulk material. Tuning the $\mathrm{THz}$ frequency by varying the pump pulse front tilt has also been recently demonstrated in a thin planar LN waveguide [89], using the additional wave guide dispersion.

\begin{tabular}{|l|l|l|l|l|l|}
\hline Pump wavelength & $800 \mathrm{~nm}$ & $800 \mathrm{~nm}$ & $800 \mathrm{~nm}$ & $1033 \mathrm{~nm}$ & $1033 \mathrm{~nm}$ \\
\hline
\end{tabular}




\begin{tabular}{|c|c|c|c|c|c|}
\hline Repetition rate & $10 \mathrm{~Hz}$ & $1 \mathrm{kHz}$ & $200 \mathrm{kHz}$ & $1 \mathrm{kHz}$ & $1 \mathrm{MHz}$ \\
\hline Pump pulse energy & $20 \mathrm{~mJ}$ & $3 \mathrm{~mJ}$ & $2.3 \mathrm{uJ}$ & $500 \mu \mathrm{J}$ & $14 \mu \mathrm{J}$ \\
\hline Pump pulse duration & $130 \mathrm{fs}$ & $80 \mathrm{fs}$ & $170 \mathrm{fs}$ & $300 \mathrm{fs}$ & $250 \mathrm{fs}$ \\
\hline THz energy & $10 \mu \mathrm{J}$ & $3 \mu \mathrm{J}$ & $400 \mathrm{pJ}$ & $100 \mathrm{~nJ}$ & $2.5 \mathrm{~nJ}$ \\
\hline THz pulse duration & $2 \mathrm{ps}$ & $1.25 \mathrm{ps}$ & $1.25 \mathrm{ps}$ & $1.25 \mathrm{ps}$ & $1.25 \mathrm{ps}$ \\
\hline THz electric field & $>250 \mathrm{kV} / \mathrm{cm}$ & $>150 \mathrm{kV} / \mathrm{cm}$ & $6 \mathrm{kV} / \mathrm{cm}$ & $30 \mathrm{kV} / \mathrm{cm}$ & $7 \mathrm{kV} / \mathrm{cm}$ \\
\hline $\mathrm{THz}$ peak intensity & $150 \mathrm{MW} / \mathrm{cm}^{2}$ & $100 \mathrm{MW} / \mathrm{cm}^{2}$ & $40 \mathrm{~kW} / \mathrm{cm} 2$ & $45 \mathrm{MW} / \mathrm{cm}^{2}$ & $60 \mathrm{~kW} / \mathrm{cm}^{2}$ \\
\hline THz average power & $100 \mu \mathrm{W}$ & $3 \mathrm{~mW}$ & $80 \mu \mathrm{W}$ & $0.5 \mathrm{~mW}$ & $0.25 \mathrm{~mW}$ \\
\hline Energy efficiency & $5 \times 10^{-4}$ & $1 \times 10^{-3}$ & $1.7 \times 10^{-4}$ & $2.5 \times 10^{-4}$ & $1.8 \times 10^{-5}$ \\
\hline $\begin{array}{l}\text { External photon } \\
\text { efficiency }\end{array}$ & $45 \%$ & $15 \%$ & $6.5 \%$ & $6 \%$ & $5.5 \%$ \\
\hline Reference & [90] & [91] & [92] & [93] & [88] \\
\hline
\end{tabular}

Table 2. Experimental results for $\mathrm{THz}$ generation using TPFP at various pump pulse repetition rates, wavelengths, and energies.

Figure 9 summarizes the scaling behavior of achievable THz output with pump pulse energy. The figure shows results obtained with various sources based on LN (with TPFP), and ZnTe. At low pulse energies, quadratic scaling is observed for both materials, as expected for a second-order process. In ZnTe saturation and linear scaling dominates in when pulse energies of $50 \mathrm{uJ}$ are exceeded. The scaling 
behavior can be described using a model from second harmonic generation theory where the efficiency $\eta$ is described as $\eta \propto \tanh ^{2}\left(\right.$ const $\left.\times \sqrt{I_{\text {opt }}}\right)$ [94]. In contrast, the scaling behavior of sLN with TPFP does not exhibit saturation [95] until very high pump pulse energies are reached. The main limiting factor in $\mathrm{THz}$ generation using ZnTe is free-carrier absorption caused by two-photon absorption of the pump light, which limits pump intensity (see next Section). Even though $\mathrm{THz}$ energies as high as $1.5 \mu \mathrm{J}$ have been demonstrated by using large pump spots [76] the achieved conversion efficiency of $1.3 \times 10^{-5}$ was rather low. In contrast, by using LN with TPFP, THz energies of $10 \mu \mathrm{J}$ [90] and $30 \mu \mathrm{J}$ [96] have been reported with pump-to-THz energy conversion efficiencies of $5 \times 10^{-4}$ and $1.1 \times 10^{-3}$, respectively. These are the most energetic ultrashort $\mathrm{THz}$ pulses ever generated from a table-top source.

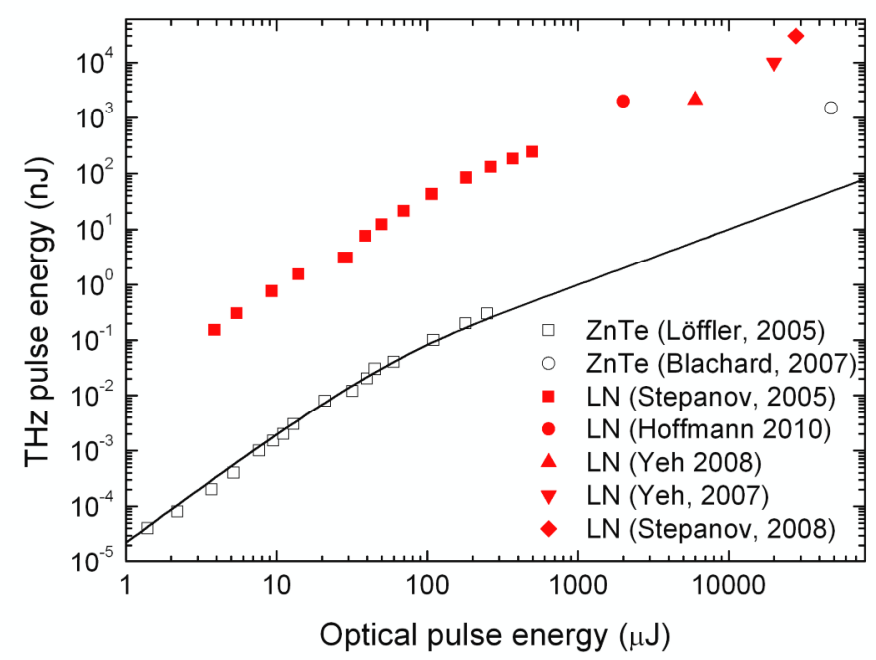

Figure 9: Scaling of the output THz pulse energy with the pump pulse energy for sources based on LN (with TPFP), and ZnTe. The solid line is a fit using model described in the text. Löffler 2005 [94], Blanchard 2007 [76], Stepanov 2005 [95], Hoffmann 2010 [97], Yeh 2008 [91], Yeh 2007 [90], Stepanov 2008 [96]. 


\subsection{Perspectives and limitations}

There is an increasing number of promising applications, which require $\mathrm{THz}$ pulse energies or field strengths even higher than the currently available level. OR in combination with TPFP is a technique with inherent scalability to higher $\mathrm{THz}$ energies or field strengths. This can be accomplished by using larger pumped areas and higher pump energies. In the following we briefly discuss perspectives and the most important limiting factors of this technique as well as possibilities to overcome some of the limitations.

THz absorption and dispersion. Infrared-active optical phonons result in absorption and dispersion of the THz radiation generated by OR. As can be seen in Table 1 the materials with the highest FOM (DAST, LN, CdTe) have relatively large absorption in the THz range, which can seriously reduce the output $\mathrm{THz}$ energy by restricting the effective material length to only a few $\mathrm{mm}$ or less. As indicated for LN [92], cooling down to cryogenic temperatures can significantly reduce the absorption coefficient. On the other hand, longer propagation distance inside the nonlinear medium leads to dispersion in the $\mathrm{THz}$ range, causing temporally stretched $\mathrm{THz}$ pulses which have a reduced $\mathrm{THz}$ peak electric field.

Multi-photon absorption. Free carriers are generated inside a nonlinear medium by multi-photon absorption of the pump radiation at high pump intensities. The increased free carrier concentration leads to increased absorption of the generated $\mathrm{THz}$ radiation, posing an upper limit on the pump intensity and leading to the saturation of the THz generation efficiency. This effect can be especially severe in the case of semiconductor materials, which have relatively small bandgaps. When using such materials for highenergy $\mathrm{THz}$ pulse generation, a possible solution is to use large crystals and large pump spot diameters 
[48]. THz pulse energies up to $1.5 \mu \mathrm{J}$ were demonstrated using a $75-\mathrm{mm}$ diameter $\mathrm{ZnTe}$ wafer and $48 \mathrm{~mJ}$ pump energy at $800 \mathrm{~nm}[76]$.

The effective lowest order of multi-photon absorption is determined by the ratio of the material bandgap to the pump photon energy. For a given material, lower-order multi-photon absorption can be efficiently suppressed by using longer pump wavelengths. For example, only three-photon absorption will be effective in semiconductors like ZnTe or GaP at $1.5 \mu \mathrm{m}$ pump wavelength, in contrast to $800-\mathrm{nm}$ or $1-\mu \mathrm{m}$ pumping where two-photon absorption is present. However, deviations from wavelengths suitable for a collinear velocity-matching requires, in general, the use of the TPFP technique for velocity matching. Due to the smaller difference between the optical group index and the THz refractive index in semiconductors (see Table 1) much smaller pulse front tilt angle (below $30^{\circ}$ is typical) is necessary for velocity matching than in LN. This also means smaller angular dispersion $\mathrm{d} \varepsilon / \mathrm{d} \lambda$, and smaller group-velocity dispersion, which is proportional to $(\mathrm{d} \varepsilon / \mathrm{d} \lambda)^{2}$ [98, 83]. As a consequence, longer effective material lengths can be used, which can, in many cases, compensate for the smaller effective nonlinearity of semiconductors [99]

Pulse-front-tilting setup. Aberrations introduced by the lens in the pulse-front-tilting setup described in Sec. 3.2 can lead to the curvature of the pump pulse front and to asymmetric THz beam profile $[99,100]$. Such distortions reduce the focusability and limit the application possibilities of the generated $\mathrm{THz}$ radiation. Such limitations can be especially severe in the case of high-energy $\mathrm{THz}$ pulses where a large pump spot is used and may prohibit further significant increase in THz pulse energy. A new compact scheme, in which no imaging optics is used as the grating is brought in contact with the nonlinear crystal has been predicted to achieve ultrashort terahertz pulses with $\mathrm{mJ}$ energy level [100] around $1 \mathrm{THz}$. The 
relatively small pulse-front-tilt angles in semiconductors are well suited for adaptation of the contactgrating technique [99]. This makes semiconductors promising candidates for further upscaling the $\mathrm{THz}$ energy and field strength in the few $\mathrm{THz}$ frequency range.

Pumping conditions. Recent numerical and experimental studies [101, 102, 99] indicate the importance of the careful choice of the pump pulse duration. Nagai et al. [102] observed enhanced THz generation at higher pump powers which was attributed to the shortening of the pump pulse due to phase modulation caused by the THz field. Based on numerical calculations [103] it is expected that THz peak electric field strengths on the $10 \mathrm{MV} / \mathrm{cm}$ scale can be generated from cryogenically cooled LN when longer ( 500 fs) pump pulses are used rather than the commonly used $~ 100$ fs. Such high field strengths in the 1-THz frequency range are anticipated to find important new applications in attosecond physics $[104,105]$ and high-field physics.

\section{Application of high field strength THz pulses}

The availability of high-field-strength ultrashort $\mathrm{THz}$ pulses enables the observation of interesting new physical phenomena such as collective vibrational excitations, the plasma frequency of a dilute free electron gas in doped semiconductors, the superconducting gap in classical BCS superconductors or Josephson plasma resonances [106]. The availability of high spectral brightness, broadband THz sources is crucial for the deployment of THz-TD-spectroscopy systems with improved signal to noise ratio for real-world applications like spatially resolved chemical recognition [107]. Fiber laser/amplifier systems have evolved rapidly during the last decade and now offer high repetition rate output in conjunction with pulse energy sufficiently high and pulse duration sufficiently short for effective THz generation while 
being economically more viable compared to conventional solid state femtosecond laser systems. The combination of improved $\mathrm{THz}$ generation efficiencies as described in the preceding sections with high pulse-energy, high repetition-rate fiber laser sources [88] will lead to improved signal to noise levels required for metrology and spectroscopic applications.

On a more fundamental level, very high intensity sources can be used to excite and to exercise coherent control over these collective degrees of freedom in the THz range. While the off-resonance excitation of dynamics with ultra short laser pulses is possible with impulsive simulated Raman scattering [108] or related techniques, a laser excitation is always accompanied by direct transitions in the optical or near infrared range which can even lead to damage in the sample. A direct, on-resonance excitation of these degrees of freedom is therefore desirable [25].

Narrowband intense $\mathrm{THz}$ sources have been available for some decades in the form gas lasers [5] and free electron lasers (FELs) [109]. Typically, gas lasers can achieve pulse durations of a few nanoseconds and because they rely on certain rotational transitions of small molecules, have only limited tunability. The first THz FELs had pulse durations of microseconds [110] while newer FELs offer temporal resolution down to tens of picoseconds while still being fairly narrowband and offering tunability over a wide frequency range [111]. Nonlinear behavior of semiconductors under strong THz excitation has been investigated using these types of sources [15] but temporal resolution was limited by long pulse durations or difficulties in synchronizing a femtosecond laser to an FEL source due to timing jitter. Optical rectification-based sources on the other hand provide sub-picosecond single- or few-cycle pulses with extremely high fields strengths and broad bandwidth. Because of their derivation from a common femtosecond laser sources, the inherent synchronization of the pump and probe pulses allow for time 
resolved experiments where the influence of the strong field on a sample can be observed directly with tens of femtosecond resolution.

\subsection{Nonresonant effects - Ponderomotive force}

In the limit of high field strengths, the interaction of an oscillating electric field with matter cannot be simply treated by a perturbative approach. In this case the interaction cannot be described by either neglecting the field aspect nor the single-quantum description. A measure for the contribution of the nonpertubative regime is the ponderomotive potential, defined by the time-averaged kinetic energy of a electron driven by the electric field. The nonperturbative regime is reached, when the ponderomotive potential of the electric field exceeds the photon energy $\hbar \omega$ or the ionization potential of the system is exceeded. For an oscillating electric field with frequency $\omega$ the ponderomotive potential is given by 
$U_{p}=\frac{e^{2} E^{2}}{4 m_{e} \omega^{2}}=\frac{2 \pi e^{2}}{m c} \frac{I}{\omega^{2}}$

where $m$ is the electron mass and $I$ is the intensity of the light. As can be seen from Equation (8) the ponderomotive potential has a quadratic dependence both on the electric field and on the inverse of the frequency. Therefore very high values of $U_{\mathrm{p}}$ can be achieved with high field strength, low frequency pulses. In laser ionization of atoms the interaction can be treated as multiphoton ionization when $U_{\mathrm{p}} \ll \hbar$ $\omega$ and as static electric field tunneling ionization when $U_{P}>I_{P}>\hbar \omega$. In the latter case they the

Keldysh parameter $\gamma=\sqrt{I_{p} / 2 U_{p}}<1$ where $I_{\mathrm{p}}$ is the ionization potential. The influence of the ponderomotive potential, i.e., effects at the transition between classical and quantum behavior, are expected to be most noticeable when $U_{\mathrm{p}} \sim \hbar \omega$, but less than or comparable to the relevant energy scale in the system like the band gap in semiconductors.

The first experiment to take advantage of the large ponderomotive potential in a single cycle $\mathrm{THz}$ pulse was the demonstration of ionization of Rydberg atoms by Jones et al [44]. Here pulses from a photoconductive source with field strengths exceeding $10 \mathrm{kV} / \mathrm{cm}$ were used to ionize sodium atoms selectively prepared by a dye laser.

\section{2. $\quad$ Nonlinear THz spectroscopy of Semiconductors}

The interaction of $\mathrm{THz}$ radiation with semiconductors has been studied extensively and include such effects as free-carrier absorption, the photon drag effect or intersubband transitions [15]. However, few studies have been reported on the nonlinear terahertz characteristics of semiconductors using high fields $[112,15,113]$ 
In semiconductors, ponderomotive effects were predicted early on [114], but strong absorption due to the band gap on the order of typically $1 \mathrm{eV}$ and the resulting high intensity damage have made such observations impossible using laser sources in the optical wavelength range where $E_{\text {photon }}$ is on the order of the band gap. By using light with significantly longer wavelength than the band gap, these problems are avoided. In the case of semiconductors, the electron mass in the ponderomotive potential is replaced by the effective mass of the carrier, thereby further increasing the value of $U_{\mathrm{p}}$. Here the case of $U_{\mathrm{p}} \sim \hbar \omega \hbar$ $\omega<E_{\text {gap }}$ can easily be achieved.

An immediate application of the large ponderomotive potential of $\mathrm{THz}$ pulses is tunneling ionization of shallow impurities. Here the energy levels of deep centers are tilted by the external electric field of the THz light so that tunneling ionization can occur [115]. This effect occurs already at comparatively low electric fields and has been observed using gas lasers. Another direct consequence of the ponderomotive potential in semiconductors is the dynamic Franz-Keldysh effect (DFKE). Here a laser field causes a deformation of the valence and conduction band wavefunction so that photon assisted tunneling is possible leading to an increased absorption at photon energies below the band gap. This effect has been demonstrated on an ultrafast time scale using mid-infrared pulses with $30 \mathrm{THz}$ frequency [116]. 
Direct acceleration of free carriers by the THz field can be observed in n-type semiconductors with high mobility. In these materials mobile free carriers form an electron gas with maximum absorption in the THz frequency range [117] at room temperature. In lightly doped GaAs, electrons can get accelerated into the ballistic regime and electron energies larger than $1 \mathrm{eV}$ can be achieved this way. The instantaneous electron velocity can far exceed the DC drift velocity at equilibrium. This is generally related to an increase in effective mass of conduction band electrons in a dispersive band structure due to the effect of nonparabolicity of the conduction band valleys at high momentum as well as intervalley transfer.

Nonlinear transmission studies in combination with modeling of the contribution of several of these effects have been reported [118] and strong absorption bleaching has been observed in a number of n-type semiconductors such as GaAs [119], InGaAs [120], Ge, Si [22] as well as in photoexcited GaAs [121]. In bulk samples, the increase in terahertz transmission can be almost one order of magnitude, making these materials suitable for use as saturable $\mathrm{THz}$ absorbers at room temperature [97].

The interaction of ballistic carriers with the optical phonon was studied in Ref. [23]. A high electric field in the terahertz range drives the Fröhlich polaron in a GaAs crystal into a highly nonlinear regime where an electron is impulsively driven from the centre of the surrounding lattice distortion. 


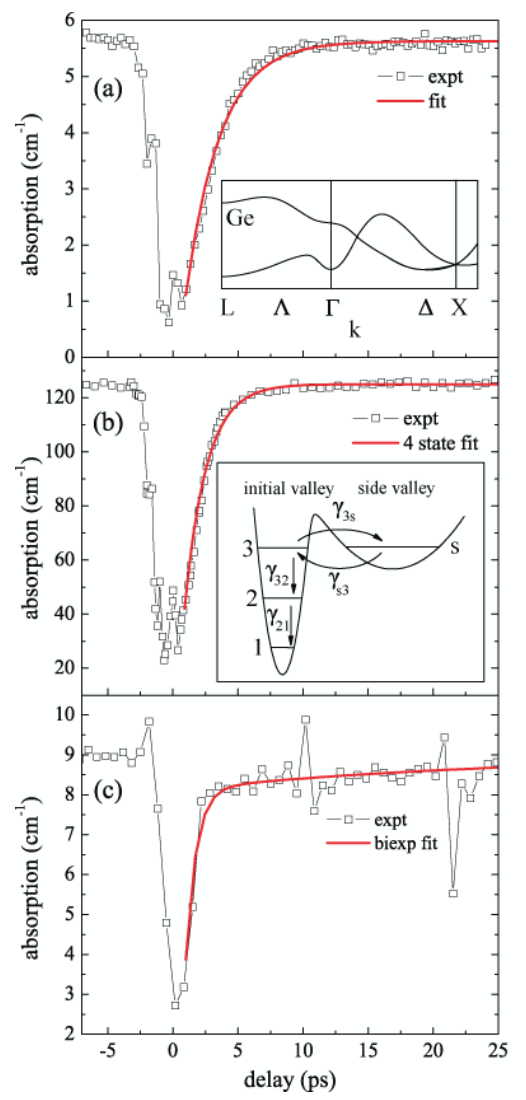

Figure 10: Dynamic behavior of the saturation of free-carrier absorption in the n-type semiconductors Ge,

GaAs and Si. Plotted is a frequency averaged (0.2-2.0 THz) absorption coefficient. The solid lines are fits using a simple rate equation model [22].

Using a THz-pump/THz probe scheme [109], the time evolution of the hot carrier distribution and the cooling can be observed [119]. Figure 10 shows the spectrally integrated THz absorption in Ge, GaAs and $\mathrm{Si}$ as function of pump-probe time delay. In all samples a strong drop in absorption is detected, with a subsequent recovery time on the order of a few picoseconds depending on the material. The dynamics can be described qualitatively by a simple model of the electron transport [121, 22]. From the spectral analysis of the data (not shown) it can be seen that the characteristics of the hot electron gas deviates 
significantly from the Drude behavior and a better theoretical modeling taking into account the hot electron properties is needed.

In the case of a low bandgap semiconductor like InSb, the energy of the electrons accelerated by the $\mathrm{THz}$ field can even exceed the bandgap, leading to pair creation of new electron hole pairs [122, 123]. Terahertz pump/Terahertz probe measurements reveal a strong increase in absorption caused by these newly generated carriers.

\subsection{THz nonlinear optics}

Classic nonlinear optical effects such as harmonic generation or self phase modulation in the $\mathrm{THz}$ range have remained largely unexplored due to lack of strong sources. Since the pioneering work of Faust and Henry using frequency mixing of an $\mathrm{H}_{2} \mathrm{O}$ laser with optical light only few experiments have been performed in which the dispersion of the nonlinear susceptibility in the $\mathrm{THz}$ range has been measured directly $[14,124]$. Intense few cycle pulses in combination with electro-optic detection should enable the study of these effects directly in the time domain.

The second-order nonlinear susceptibility in non-centrosymmetric crystals has two contributions: the (bound) electrons and the ion cores [125]. This is generally expressed as

$$
\chi^{2}\left(\omega_{1}-\omega, \omega, \omega_{1}\right)=\chi_{E}^{2}\left[1+C_{1} \frac{1}{D(\omega)}\right]
$$

with $D(\omega)=1-\frac{\omega}{\omega_{T O}^{2}}-\frac{i \omega \Gamma}{\omega_{T O}^{2}}$ where $\omega_{\mathrm{TO}}$ is the TO phonon frequency and $\Gamma$ the damping constant and $\chi^{(2)} \mathrm{E}$ is the electronic part of the nonlinear susceptibility. The Faust-Henry coefficient $C_{1}$ expresses the ratio between the lattice-induced and the electronic contributions to the nonlinear coefficient. In ferroelectric 
materials like $\mathrm{LiTaO}_{3}$ and $\mathrm{LiNbO}_{3}$, the contribution of the ionic part to $\chi^{(2)}$ can be an order of magnitude larger than the electronic part [126]. Direct observation of second harmonic generation in $\mathrm{LiTaO}_{3}$ has been reported by Mayer [13] using 40 ns pulse duration gas laser in combination with steep filters in order to suppress the fundamental. A similar experiment in GaAs using a free electron laser at wavelength in the range of 3.5 to $6 \mathrm{THz}$ has been carried out by Dekorsy et al [14]. Using table top time-domain methods, second harmonic generation in lithium niobate has been observed in the focus of two intense phonon-polaritons in a waveguide [127].

Strong terahertz fields can also induce polarization in nonpolar media, leading to transient birefringence, which can then be probed by an optical probe pulse. This is analogous to the optical Kerr effect, and involves a third order $\left(\chi^{(3)}\right)$ nonlinear process combining the action of an intense THz field onto the polarization state of a probe pulse at optical frequencies. Figure 11a shows the induced birefringence in four different organic solvents as function of time compared with the applied single-cycle THz field [24]. The signal is composed of two contributions, a fast electronic response that follows the square of the $\mathrm{THz}$ field directly, and a slower, exponential decay resulting from subsequent relaxationof the partially aligned molecules in the liquid which is on the order of a few ps. The overall birefringence of the aligned molecule scales quadratically with the peak THz field (Figure 11b). 

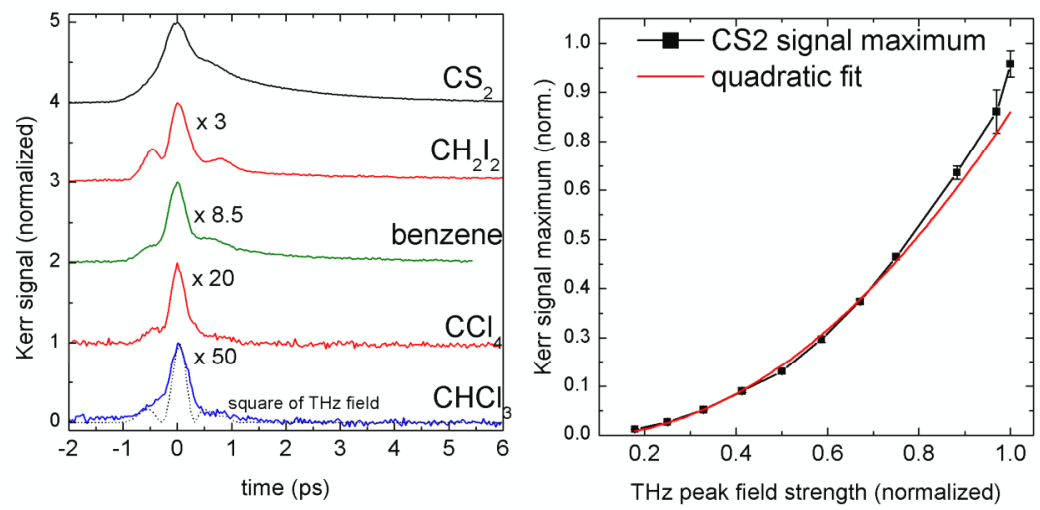

Figure 11: (a) Terahertz-induced birefringence in polarizable liquids. In the time domain, the signal follows the square of the THz pulse. (b) The peak signal scales quadratically with the THz field strength, demonstrating the $\chi^{(3)}$ nature. From [24]

\subsection{Vibrational excitation}

Coherent control over vibrational degrees of freedom using laser pulses has been a long standing goal in ultrafast spectroscopy. Molecular or ionic motions in the $\mathrm{THz}$ frequency range are a promising target for coherent control since they are in certain systems directly connected to macroscopic properties such as ferroelectricity or ferroelasticity. Large-amplitude THz motion can be excited by optical ultrashort pulse through the impulsive stimulated Raman scattering process and controlled access to the anharmonic regime of the lattice potential along the soft mode has been demonstrated [128]. However, excitation with optical pulses is limited to Raman active modes and the high excitation fluencies necessary in these experiments can lead to damage in opaque samples.

The quantum mechanical analog to the creation of large amplitude motion in an oscillator is ladder climbing. This is achieved by a strong field applied over a time shorter than the vibrational period of the oscillator. Since most realistic vibrational modes relevant for chemical dynamics are anharmonic, the 
application of a broadband pulse is more efficient than a strictly monochromatic multi-cycle pulse. Ladder climbing in a phonon mode of the crystalline amino acid excited by a single cycle $\mathrm{THz}$ pulse has been reported recently by Jewariya et al. [129]. In amino acids and other hydrogen bonded crystalline substances like sugars, the dielectric properties in the THz range are determined by low-lying vibrations which are an admixture of inter-and intramolecular degrees of freedom. These modes can exhibit a large anharmonicity [130] which can be exploited by a series of coherent transitions between quantum levels excited by a strong THz pulse.

\section{Conclusion}

Today tabletop generation of ultrashort $\mathrm{THz}$ pulses with energies up to several microjoules and field levels on the order of $1 \mathrm{MV} / \mathrm{cm}$ at $1 \mathrm{THz}$ frequency is now possible in a routine and reliable manner.

Photoconductive switches based on semiconductors are an efficient method of THz generation for comparatively low laser fluencies. Due to strong saturation effects with pump pulse intensity, scaling to $\mathrm{THz}$ energies above the micro-Joule level is generally limited.

THz generation from gas plasmas produces single cycle pulses with moderately high peak fields and extremely broad bandwidth, limited only by laser pulse duration and detection bandwidth. These pulses are an ideal probe for pump-probe spectroscopy of ultrafast processes in solids.

Nonlinear optical processes like OR and difference frequency generation of femtosecond laser pulses are versatile techniques for single-cycle as well as tunable $\mathrm{THz}$ pulse generation. Using the technique of tilted-pulse-front pumping for OR of femtosecond pulses in lithium niobate, extremely high THz pulse energies (on the 10- $\mu \mathrm{J}$ scale) and pump-to-THz conversion efficiencies have been demonstrated. The 
technique of TPFP is inherently scalable by using higher pump energies and larger pumped areas allowing for further increasing the available $\mathrm{THz}$ pulse energy and field strength.

Single-cycle THz pulses with high field strengths are an ideal tool to directly study the interaction of strong electric fields with matter at ultrafast timescales. The area of nonlinear THz spectroscopy is still at an early stage, but the prospects for spectroscopy of and control over collective degrees of freedom such as motions of dipoles and ions as well as electronic correlations are becoming increasingly clear.

\section{Acknowledgements}

The authors acknowledge helpful discussion with János Hebling and Mark D. Thomson and Andrea Cavalleri. MCH acknowledges funding from the Max Planck society. JAF acknowledges financial support from Hungarian Scientific Research Fund (OTKA), grant numbers 76101 and 78262, and from Science, Please! Research Team on Innovation (SROP-4.2.2/08/1/2008-0011). 


\section{References}

[1] Harwit M 2004 The Herschel mission Advances in Space Research 34 568-72

[2] Glover RE and Tinkham M 1956 Transmission of Superconducting Films at MillimeterMicrowave and Far Infrared Frequencies Physical Review 104844

[3] Kimmit M F 2003 Restrahlen to T-Rays - 100 Years of Terahertz Radiation Journal of Biological Physics 29 77-85

[4] Hadni A 2003 A Short History of 50 Years of Research in the Far Infrared: 1952-2002 International Journal of Infrared and Millimeter Waves 24 91-118

[5] Chang T Y, Bridges T J and Burkhardt E G $1970 \mathrm{cw}$ Submillimeter Laser Action in Optically Pumped Methyl Fluoride, Methyl Alcohol, and Vinyl Chloride Gases Applied Physics Letters 17 249-51

[6] Mattauch R J and Crowe T W 1987 GaAs Schottky Devices for Submillimeter Wavelengths Int. J. Infrared and Millim. Waves 8 1235-41,

[7] Sirtori C, Dhillon S, Faugeras C, Vasanelli A and Marcadet X 2006 Quantum cascade lasers: the semiconductor solution for lasers in the mid- and far-infrared spectral regions Phys. Status Solidi A 203 3533-7

[8] Kohler R, Tredicucci A, Beltram F, Beere H E, Linfield E H, Davies A G, Ritchie D A, lotti R C and Rossi F 2002 Terahertz semiconductor-heterostructure laser Nature 417 156-9

[9] Kozlov G and Volkov A 1998 Millimeter and Submillimeter Wave Spectroscopy of Solids, ed G Grüner (Berlin Heidelberg: Springer)

[10] Bründermann E, Röser H P and Kimmitt M F 1993 Tunable p-germanium monocrystal laser for the wavelength region from $70 \mu \mathrm{m}$ to $350 \mu \mathrm{m}$ Laser und Optoelektronik 25 48-60

[11] Andronov A A, Nozdrin Y N and Shastin V N 1987 Tunable FIR lasers in semiconductors using hot holes Infrared Physics 27 31-8

[12] Horvat J and Lewis R A 2009 Peeling adhesive tape emits electromagnetic radiation at terahertz frequencies Opt. Lett. 34 2195-7

[13] Mayer A and Keilmann F 1986 Far-infrared nonlinear optics. I. $\chi(2)$ near ionic resonance Phys. Rev. B 226954

[14] Dekorsy T, Yakovlev V A, Seidel W, Helm M and Keilmann F 2003 Infrared-Phonon-Polariton Resonance of the Nonlinear Susceptibility in GaAs Phys. Rev. Lett. 90055508

[15] Ganichev S and Prettl W 2005 Intense Terahertz Excitation of Semiconductors: Oxford Univ Press)

[16] Morris J R and Shen Y R 1971 Far-infrared generation by picosecond pulses in electro-optical materials Opt. Commun. 3 81-4

[17] Auston D H and Smith P R 1983 Generation and detection of millimeter waves by picosecond photoconductivity Appl. Phys. Lett. 43 631-3

[18] Fattinger C and Grischkowsky D 1989 Terahertz Beams Appl. Phys. Lett. 54 490-2

[19] Auston D H, Cheung K P, Valdmanis J A and Kleinman D A 1984 Cherenkov Radiation from Femtosecond Optical Pulses in Electro-Optic Media Phys. Rev. Lett. 53 1555-8

[20] Schmuttenmaer C A 2004 Exploring dynamics in the far-infrared with terahertz spectroscopy Chemical Reviews 104 1759-79 
[21] Huber R, Tauser F, Brodschelm A, Bichler M, Abstreiter G and Leitenstorfer A 2001 How manyparticle interactions develop after ultrafast excitation of an electron-hole plasma Nature $\mathbf{4 1 4}$ 286-9

[22] Hebling J, Hoffmann M C, Hwang H Y, Yeh K-L and Nelson K A 2010 Observation of nonequilibrium carrier distribution in $\mathrm{Ge}, \mathrm{Si}$, and $\mathrm{GaAs}$ by terahertz pump--terahertz probe measurements Physical Review B $\mathbf{8 1} 035201$

[23] Gaal P, Kuehn W, Reimann K, Woerner M, Elsaesser T and Hey R 2007 Internal motions of a quasiparticle governing its ultrafast nonlinear response Nature 450 1210-3

[24] Hoffmann M C, Brandt N C, Hwang H Y, Yeh K-L and Nelson K A 2009 Terahertz Kerr effect Applied Physics Letters 95 231105-3

[25] Rini M, Tobey R a, Dean N, Itatani J, Tomioka Y, Tokura Y, Schoenlein R W and Cavalleri A 2007 Control of the electronic phase of a manganite by mode-selective vibrational excitation Nature $44972-4$

[26] Jameson A D, Tomaino J L, Lee Y S, Prineas J P, Steiner J T, Kira M and Koch S W 2009 Transient optical response of quantum well excitons to intense narrowband terahertz pulses Applied Physics Letters 953

[27] Reimann K 2007 Table-top sources of ultrashort THz pulses Reports on Progress in Physics 70 1597-632

[28] Dexheimer S 2008 Terahertz Spectroscopy (Boca Raton: CRC Press)

[29] Mittleman D 2004 Sensing with Terahertz Radiation vol 85 (Berlin: Springer)

[30] You D, Jones R R, Bucksbaum P H and Dykaar D R 1993 Generation of high-power sub-singlecycle 500-fs electromagnetic pulses Opt. Lett. 18 290-2

[31] Lochtefeld A J, Melloch M R, Chang J C P and Harmon E S 1996 The role of point defects and arsenic precipitates in carrier trapping and recombination in low-temperature grown GaAs Appl. Phys. Lett. 69 1465-7

[32] Loka H S, Benjamin S D and Smith P W E 1998 Optical characterization of low-temperaturegrown GaAs for ultrafast all-optical switching devices IEEE Jounal of Quantum Electronics 34 1426-37

[33] Harmon E S, Melloch M R, Woodall J M, Nolte D D, Otsuka N and Chang C L 1993 Carrier lifetime versus anneal in low temperature growth GaAs Appl. Phys. Lett. 63 2248-50

[34] Gregory I S, Baker C, Tribe W R, Evans M J, Beere H E, Linfield E H, Davies A G and Missous M 2003 High resistivity annealed low-temperature GaAs with 100 fs lifetimes Appl. Phys. Lett. 83 4199-201

[35] Prasankumar R P, Scopatz A, Hilton D J, Taylor A J, Averitt R D, Zide J M and Gossard A C 2005 Carrier dynamics in self-assembled ErAs nanoislands embedded in GaAs measured by opticalpump terahertz-probe spectroscopy Applied Physics Letters 86 201107-3

[36] Winnerl S, Peter F, Nitsche S, Dreyhaupt A, Zimmermann B, Wagner M, Schneider H, Helm M and Kohler $\mathrm{K} 2008$ Generation and detection of $\mathrm{THz}$ radiation with scalable antennas based on GaAs substrates with different carrier lifetimes IEEE J. Sel. Top. Quantum Electron. 14 449-57

[37] Auston D H, Cheung K P and Smith P R 1984 Picosecond photoconducting Hertzian dipoles Applied Physics Letters 45 284-6

[38] Sartorius B, Roehle H, Künzel H, Böttcher J, Schlak M, Stanze D, Venghaus H and Schell M 2008 All-fiber terahertz time-domain spectrometer operating at $1.5 \mu \mathrm{m}$ telecom wavelengths Opt. Express 16 9565-70 
[39] Shen Y C, Upadhya P C, Linfield E H, Beere H E and Davies A G 2003 Ultrabroadband terahertz radiation from low-temperature-grown GaAs photoconductive emitters Applied Physics Letters 83 3117-9

[40] Sakai K 2005 Terahertz Optoelectronics (Springer)

[41] Stephen E R and Grischkowsky D 1991 Trap-enhanced electric fields in semi-insulators: The role of electrical and optical carrier injection Appl. Phys. Lett. 59 1972-4

[42] Pfeifer T, Heiliger H-M, Loffler T, Ohlhoff C, Meyer C, Lupke G, Roskos H G and Kurz H 1996 Optoelectronic on-chip characterization of ultrafast electric devices: Measurement techniques and applications IEEE Journal on Selected Topics in Quantum Electronics 2 586-604

[43] Benicewicz P K, Roberts J P and Taylor A J 1994 Scaling of terahertz radiation from largeaperture biased photoconductors J. Opt. Soc. Am. B 11 2533-46

[44] Jones R R, You D and Bucksbaum P H 1993 Ionization of Rydberg atoms by subpicosecond halfcycle

electromagnetic pulses Phys. Rev. Lett. 70 1236-9

[45] Dreyhaupt A, Winnerl S, Dekorsy T and Helm M 2005 High-intensity terahertz radiation from a microstructured

large-area photoconductor Appl. Phys. Lett. 86 121114-6

[46] Beck M, Schafer H, Klatt G, Demsar J, Winnerl S, Helm M and Dekorsy T 2010 Impulsive terahertz radiation with high electric fields from an amplifier-driven large-area photoconductive antenna Optics Express 18 9251-7

[47] Dreyhaupt A, Winnerl S, Helm M and Dekorsy T 2006 Optimum excitation conditions for the generation of high-electric-field terahertz radiation from an oscillator-driven photoconductive device Opt. Lett. 31 1546-8

[48] Löffler T, Hahn T, Thomson M, Jacob F and Roskos H 2005 Large-area electro-optic ZnTe terahertz emitters Optics Express 13 5353-62

[49] Thomson M D, Kreß M, Löffler T and Roskos H G 2007 Broadband THz emission from gas plasmas induced by femtosecond optical pulses: From fundamentals to applications Laser Photon. Rev. 1349

[50] Karpowicz N, Lu X and Zhang X-C 2009 Terahertz gas photonics Journal of Modern Optics 56 1137-50

[51] Hamster H, Sullivan A, Gordon S, White W and Falcone R W 1993 Subpicosecond, electromagnetic pulses from intense laser-plasma interaction Physical Review Letters 712725

[52] Cook D J and Hochstrasser R M 2000 Intense terahertz pulses by four-wave rectification in air Opt. Lett. 25 1210-2

[53] Kim K Y, Taylor A J, Glownia J H and Rodriguez G 2008 Coherent control of terahertz supercontinuum generation in ultrafast laser-gas interactions Nature Photon. 2 605-9

[54] Thomson M D, Blank V and Roskos H G 2010 Terahertz white-light pulses from an air plasma photo-induced by incommensurate two-color optical fields Optics Express 18 23173-82

[55] Dai J M, Karpowicz N and Zhang X C 2009 Coherent Polarization Control of Terahertz Waves Generated from Two-Color Laser-Induced Gas Plasma Physical Review Letters 1034

[56] Rodriguez G and Dakovski G L 2010 Scaling behavior of ultrafast two-color terahertz generation in plasma gas targets: energy and pressure dependence Opt. Express 18 15130-43

[57] Kress M, Löffler T, Eden S, Thomson M and Roskos H G 2004 Terahertz-pulse generation by photoinoization of air with laser pulses composed of both fundamental and second-harmonic waves Opt. Lett. 29 
[58] Kress M, Loffler T, Thomson M D, Dorner R, Gimpel H, Zrost K, Ergler T, Moshammer R, Morgner $\mathrm{U}$, Ullrich J and Roskos H G 2008 Few-cycle laser pulses: The carrier-envelope phase, its role in the THz emission from laser-generated plasmas and a new way to measure it Acta Phys. Pol. A $113769-76$

[59] Kim K-Y, Glownia J H, Taylor A J and Rodriguez G 2007 Terahertz emission from ultrafast ionizing air in symmetry-broken laser fields Opt. Express 2007 4577-84

[60] Karpowicz N and Zhang X C 2009 Coherent Terahertz Echo of Tunnel Ionization in Gases Physical Review Letters 102 093001-4

[61] Babushkin I, Kuehn W, Köhler C, Skupin S, Bergé L, Reimann K, Woerner M, Herrmann J and Elsaesser T 2010 Ultrafast Spatiotemporal Dynamics of Terahertz Generation by lonizing TwoColor Femtosecond Pulses in Gases Physical Review Letters 105053903

[62] Dai J, Xie X and Zhang X-C 2006 Detection of Broadband Terahertz Waves with a Laser-Induced Plasma in Gases Phys. Rev. Lett. 97103903

[63] Karpowicz N, Dai J, Lu X, Chen Y, Yamaguchi M, Hongwei Zhao H, Zhang X-C, Zhang L, Zhang C, Price-Gallagher M, Fletcher C, Mamer O, Lesimple A and Johnson K 2008 Coherent heterodyne time-domain spectrometry covering the entire "terahertz gap" Appl. Phys. Lett. 92011131

[64] Liu J, Dai J, Chin S L and Zhang X C 2010 Broadband terahertz wave remote sensing using coherent manipulation of fluorescence from asymmetrically ionized gases Nat Photon 4 627-31

[65] Bass M, Franken P A, Ward J F and Weinreich G 1962 Optical Rectification Phys. Rev. Lett. 9446 8

[66] Yang K H, Richards P L and Shen Y R 1971 Generation of Far-Infrared Radiation by Picosecond Light Pulses in LiNbO3 Appl. Phys. Lett. 19 320-3

[67] Hu B B, Zhang X-C and Auston D H 1990 Free-space radiation from electrooptic crystals Appl. Phys. Lett. 56 506-8

[68] Vodopyanov K L 2006 Optical generation of narrow-band terahertz packets in periodically inverted electro-optic crystals: conversion efficiency and optimal laser pulse format Optics Express 14 2263-76

[69] Sutherland R L 2003 Handbook of Nonlinear Optics (New York: Marcel Dekker, Inc.)

[70] Wynne K and Carey J J 2005 An integrated description of terahertz generation through optical rectification, charge transfer, and current surge Opt. Commun. 256 400-13

[71] Hebling J, Yeh K-L, Hoffmann M C, Bartal B and Nelson K A 2008 Generation of high-power terahertz pulses by tilted-pulse-front excitation and their application possibilities J. Opt. Soc. Am. B 25 B6-B19

[72] Schneider A, Stillhart M and Günter P 2006 High efficiency generation and detection of terahertz pulses using laser pulses at telecommunication wavelengths Opt. Express 14 5376-84

[73] Shibuya T, Akiba T, Suizu K, Uchida H, Otani C and Kawase K 2008 Terahertz-wave generation using a 4-dimethylamino- $\mathrm{N}$-methyl-4-stilbazolium tosylate crystal under intra-cavity conditions Appl. Phys. Express 13

[74] Miyamoto K, Minamide H, Fujiwara M, Hashimoto $\mathrm{H}$ and Ito $\mathrm{H} 2008$ Widely tunable terahertzwave generation using an N-benzyl-2-methyl-4-nitroaniline crystal Opt. Lett. 33 252-4

[75] Hunziker C, Kwon S-J, Figi H, Juvalta F, Kwon O-P, Jazbinsek M and Günter P 2008 Configurationally locked, phenolic polyene organic crystal 2-\{3-(4-hydroxystyryl)-5,5dimethylcyclohex-2-enylidene\}malononitrile: linear and nonlinear optical properties J. Opt. Soc. Am B 25 1678-83 
[76] Blanchard F, Razzari L, Bandulet H C, Sharma G, Morandotti R, Kieffer J C, Ozaki T, Reid M, Tiedje H F, Haugen H K and Hegmann F A 2007 Generation of $1.5 \mu \mathrm{J}$ single-cycle terahertz pulses by optical rectification from a large aperture ZnTe crystal Optics Express 15 13213-20

[77] Huber R, Brodschelm A, Tauser F and Leitenstorfer A 2000 Generation and field-resolved detection of femtosecond electromagnetic pulses tunable up to $41 \mathrm{THz}$ Applied Physics Letters 76 3191-3

[78] Reimann K, Smith R P, Weiner A M, Elsaesser T and Woerner M 2003 Direct field-resolved detection of terahertz transients with amplitudes of megavolts per centimeter Opt. Lett. 28 471-3

[79] Manzoni C, Foerst M, Ehrke H and Cavalleri A 2010 Single-Shot detection and direct control of carrier phase drift of mid-IR pulses Optics Letters 35 757-9

[80] Barker A S and Loudon R 1972 Response Functions in the Theory of Raman Scattering by Vibrational and Polariton Modes in Dielectric Crystals Reviews of Modern Physics 4418

[81] Lee Y-S, Meade T, Perlin V, Winful H and Norris T B 2000 Generation of narrow-band terahertz radiation via optical rectification of femtosecond pulses in periodically poled lithium niobate Appl. Phys. Lett. 76 2505-7

[82] Hebling J, Almasi G, Kozma I and Kuhl J 2002 Velocity matching by pulse front tilting for large area $\mathrm{THz}$-pulse generation Optics Express 10 1161-6

[83] Hebling J 1996 Derivation of the pulse front tilt caused by angular dispersion Opt. Quantum Electron. 28 1759-63

[84] Martinez E O 1986 Pulse distortions in tilted pulse schemes for ultrashort pulses Opt. Commun. 59 229-32

[85] Kleinman D and Auston D 1984 Theory of electrooptic shock radiation in nonlinear optical media IEEE Journal of Quantum Electronics 20 964- 70

[86] Auston D H 1983 Subpicosecond electro-optic shock waves Appl. Phys. Lett. 43 713-5

[87] KoehI R M and Nelson K A 2001 Coherent optical control over collective vibrations traveling at lightlike speeds J. Chem. Phys. 114 1443-6

[88] Hoffmann M C, Yeh K-L, Hwang H, Y. , Sosnowski T S, Prall B S, Hebling J and Nelson K A 2008 Fiber laser pumped high average power single-cycle terahertz pulse source Appl. Phys. Lett. 93 141107

[89] Lin K H, Werley C A and Nelson K A 2009 Generation of multicycle terahertz phonon-polariton waves in a planar waveguide by tilted optical pulse fronts Applied Physics Letters 953

[90] Yeh K L, Hoffmann M C, Hebling J and Nelson K A 2007 Generation of $10 \mu \mathrm{J}$ ultrashort terahertz pulses by optical rectification Appl. Phys. Lett. 90171121

[91] Yeh K-L, Hebling J, Hoffmann M C and Nelson K A 2008 Generation of high average power $1 \mathrm{kHz}$ shaped THz pulses via optical rectification Optics Commun. 281 3567-70

[92] Hebling J, Stepanov A G, Almási G, Bartal B and Kuhl J 2004 Tunable THz pulse generation by optical rectification of ultrashort laser pulses with tilted pulse fronts Applied Physics B: Lasers and Optics 78 593-9

[93] Hoffmann M C, Yeh K-L, Hebling J and Nelson K A 2007 Efficient terahertz generation by optical rectification at $1035 \mathrm{~nm}$ Optics Express 15 11706-13

[94] Löffler T, Kreß M, Thomson M, Hahn T, Hasegawa N and G. R H 2005 Comparative performance of terahertz emitters in amplifier-laser-based systems Semicond. Sci. Technol. 20 S134-S41

[95] Stepanov A G, Kuhl J, Kozma I Z, Riedle E, Almási G and Hebling J 2005 Scaling up the energy of THz pulses created by optical rectification Optics Express 135762 
[96] Stepanov A G, Bonacina L, Chekalin S V and Wolf J-P 2008 Generation of $30 \mu \mathrm{J}$ single-cycle terahertz pulses at $100 \mathrm{~Hz}$ repetition rate by optical rectification Opt. Lett. 33 2497-9

[97] Hoffmann M C and Turchinovich D 2010 Semiconductor saturable absorbers for ultrafast terahertz signals Applied Physics Letters 963

[98] Martínez O E, Gordon J P and Fork R L 1984 Negative group-velocity dispersion using refraction J. Opt. Soc. Am. A 1 1003-6

[99] Fülöp J A, Pálfalvi L, Almási G and Hebling J 2010 Design of high-energy THz sources based on optical rectification Opt. Express 18 12311-27

[100] Pálfalvi L, Fülöp J A, Almási G and Hebling J 2008 Novel setups for extremely high power singlecycle terahertz pulse generation by optical rectification Appl. Phys. Lett. 92171107

[101] Bakunov M I, Bodrov S B and Tsarev M V 2008 Terahertz emission from a laser pulse with tilted front: Phase-matching versus Cherenkov effect J. Appl. Phys. 104, 073105 (2008); DOI:10.1063/1.2989999 104073105

[102] Nagai M, Jewariya M, Ichikawa Y, Ohtake H, Sugiura T, Uehara Y and Tanaka K 2009 Broadband and high power terahertz pulse generation beyond excitation bandwidth limitation via $\chi(2)$ cascaded processes in $\mathrm{LiNbO}_{3}$ Opt. Express 17 11543-9

[103] Fülöp J A, Pálfalvi L and Hebling J Towards mJ-level ultrafast THz pulses.

[104] Hong W, Lu P, Lan P, Zhang Q and Wang X 2009 Few-cycle attosecond pulses with stabilizedcarrier-envelope phase in the presence of a strong terahertz field Optics Express 17 5139-46

[105] Balogh E, Fülöp J A, Hebling J, Dombi P, Farkas G and Varjú K 2010 Attosecond pulse generation in noble gases in the presence of extreme high intensity THz pulses. In: 31st European Conference on Laser Interaction with Matter (XXXI ECLIM), (Budapest, Hungary

[106] Thorsmølle V K, Averitt R D, Maley M P, Hundley M F, Koshelev A E, Bulaevskii L N and Taylor A J 2002 Evidence for linelike vortex liquid phase in $\mathrm{Tl}_{2} \mathrm{Ba}_{2} \mathrm{CaCu}_{2} \mathrm{O}_{8}$ probed by the Josephson plasma resonance Physical Review B 66012519

[107] Fischer B, Hoffmann M, Helm H, Modjesch G and Jepsen P U 2005 Chemical recognition in terahertz time-domain spectroscopy and imaging Semicond. Sci. Technol. 20 S246-S53

[108] Dhar L, Rogers J A and Nelson K A 1997 Time-resolved vibrational spectroscopy in the impulsive limit Chem. Rev. 94 157-93

[109] van der Meer A F G 2004 FELS, nice toys or efficient tools? Nucl. Instrum. Methods Phys. Res. Sect. A-Accel. Spectrom. Dect. Assoc. Equip. 528 8-14

[110] Elias L R 1987 Free-Electron Laser Research at the University of California, Santa Barbara IEEE J. Quantum Electron. 23 1470-5

[111] Michel P, Gabriel F, Grosse E, Evtushenko P, Dekorsy T, Krenz M, Helm M, Lehnert U, Seidel W and Wünsch R 2004 First lasing of the ELBE mid-IR FEL. In: Proceedings of the 26th International FEL Conference, ed R e Bakker, et al. (Trieste: Comitato Conferenze Elettra) p 8

[112] S. D. Ganichev J D, and W. Prettl, 1994 Nonlinear far-infrared absorption in InSb due to light impact ionization Appl. Phys. Lett. 64 1977-80

[113] Wagner M, Schneider H, Stehr D, Winnerl S, Andrews A M, Schartner S, Strasser G and Helm M 2010 Observation of the Intraexciton Autler-Townes Effect in GaAs/AIGaAs Semiconductor Quantum Wells Physical Review Letters 105167401

[114] Yacoby Y 1968 High-Frequency Franz-Keldysh Effect Physical Review 169610

[115] Ganichev S D, Yassievich I N and Prettl W 2002 Tunnelling ionization of deep centres in highfrequency electric fields J. Phys.-Condes. Matter 14 R1263-R95 
[116] Chin A H, Bakker J M and Kono J 2000 Ultrafast electro-absorption at the transition between classical and quantum response Phys. Rev. Lett. 853293

[117] Dressel M and Grüner G 2002 Electrodynamics of Solids (Cambridge: Cambridge University Press)

[118] Kuehn W, Gaal P, Reimann K, Woerner M, Elsaesser T and Hey R 2010 Coherent Ballistic Motion of Electrons in a Periodic Potential Physical Review Letters 104146602

[119] Hoffmann M C, Hebling J, Hwang H Y, Yeh K L and Nelson K A 2009 THz-pump/THz-probe spectroscopy of semiconductors at high field strengths [Invited] J. Opt. Soc. Am. B-Opt. Phys. 26 A29-A34

[120] Razzari L, Su F H, Sharma G, Blanchard F, Ayesheshim A, Bandulet H C, Morandotti R, Kieffer J C, Ozaki T, Reid M and Hegmann F A 2009 Nonlinear ultrafast modulation of the optical absorption of intense few-cycle terahertz pulses in $\mathrm{n}$-doped semiconductors Physical Review $B \mathbf{7 9} 4$

[121] Su F H, Blanchard F, Sharma G, Razzari L, Ayesheshim A, Cocker T L, Titova L V, Ozaki T, Kieffer J C, Morandotti R, Reid M and Hegmann F A 2009 Terahertz pulse induced intervalley scattering in photoexcited GaAs Optics Express 17 9620-9

[122] Wen H, Wiczer M and Lindenberg A M 2008 Ultrafast electron cascades in semiconductors driven by intense femtosecond terahertz pulses Phys. Rev. B 78125203

[123] Hoffmann M C, Hebling J, Hwang H Y, Yeh K L and Nelson K A 2009 Impact ionization in InSb probed by THz-pump THz-probe spectroscopy Phys. Rev. B 79 161201(R)

[124] Keilmann F 1991 Nonlinear Far-Infrared Spectroscopy of Solids Infrared Phys 31 373-80

[125] Faust W L and Henry C H 1966 Mixing of visible and near-resonance infrared light in GaP Phys. Rev. Lett. 17 1265-8

[126] Boyd G D and Pollack M A 1973 Microwave Nonlinearities in Anisotropic Dielectrics and Their Relation to Optical and Electro-Optical Nonlinearities Phys. Rev. B 75345 - 59

[127] Hornung T, Yeh K L and Nelson K A 2006 Terahertz Nonlinear Response in Lithium Niobate. In: 15th International Conference on Ultrafast Phenomena: (Optical Society of America) p MG8

[128] Brennan C J and Nelson K A 1997 J. Chem. Phys. 1079691

[129] Jewariya M, Nagai M and Tanaka K 2010 Ladder climbing on the anharmonic intermolecular potential in an amino acid microcrystal via an intense monocycle terahertz pulse Phys. Rev. Lett. (accepted)

[130] Walther M, Fischer B M and Jepsen P U 2003 Chem. Phys. 288261 